Supporting Information

\title{
MsDBP: Exploring DNA-Binding Proteins by Integrating Multiscale Sequence Information via Chou's Five-step Rule
}

\author{
Xiuquan Du, ${ }^{*}$, Yanyu Diao ${ }^{1}$, Heng Liu' ${ }^{2}$, Shuo $\mathrm{Li}^{3}$ \\ ${ }^{1}$ The School of Computer Science and Technology, Anhui University, Hefei, Anhui, \\ China \\ ${ }^{2}$ Department of Gastroenterology, The First Affiliated Hospital of Anhui Medical \\ University, Hefei, Anhui, China \\ ${ }^{3}$ Department of Medical Imaging, Western University, London, ON N6A 3K7, \\ Canada.
}

*E-mail: dxqllp@163.com. Phone: +086-13721058041

Table of Contents

Table S1. Predictive results of MsDBP on DBP2858 dataset.

Figure S1. Parameter settings for the model trained on reduced PDB1075.

Session S1. Read me_Source code and dataset. 
Table S1. Predictive results of MsDBP on DBP2858 dataset. The DBP2858 dataset contains 1049 DBPs of the human, 929 DBPs of the A.thaliana, 424 DBPs of the mouse, 314 DBPs of the S.cerevisiae and 142 DBPs of the fruit fly, respectively.

\begin{tabular}{|c|c|c|c|c|c|c|c|c|}
\hline Human & & & & & & & & \\
\hline pro_id & pred_label & proba & pro_id & pred_label & proba & pro_id & pred_label & proba \\
\hline P04637 & 0 & 0.005522 & P17844 & 0 & $2.43 E-05$ & P14735 & 1 & 0.999745 \\
\hline P23511 & 1 & 0.99997 & Q92963 & 0 & $7.09 E-05$ & P78363 & 0 & 1.19E-05 \\
\hline Q53GQ0 & 0 & $2.98 \mathrm{E}-06$ & Q92985 & 1 & 0.999994 & Q96PY6 & 1 & 0.999974 \\
\hline Q9Y572 & 1 & 0.999848 & P27694 & 1 & 0.999177 & Q9Y394 & 0 & 0.073929 \\
\hline O75874 & 0 & $1.71 \mathrm{E}-05$ & P51955 & 1 & 0.999994 & P52198 & 1 & 0.999815 \\
\hline Q9P2D1 & 1 & 0.999993 & Q8N5I4 & 0 & $2.75 E-06$ & P38919 & 1 & 0.998734 \\
\hline Q13469 & 1 & 0.999954 & 075116 & 0 & $9.89 E-05$ & P13569 & 0 & 0.045184 \\
\hline Q8IY37 & 1 & 0.999852 & O15111 & 1 & 0.999815 & Q9NPP4 & 1 & 0.678476 \\
\hline P51449 & 1 & 0.999987 & Q16539 & 0 & $1.34 \mathrm{E}-05$ & Q9C098 & 1 & 0.999754 \\
\hline P06213 & 0 & 0.0042 & Q7RTR0 & 0 & 0.000161 & P61587 & 1 & 0.999988 \\
\hline P10275 & 1 & 0.998472 & Q9BTZ2 & 0 & 4.62E-05 & P78415 & 1 & 0.99999 \\
\hline Q12857 & 1 & 0.999987 & Q04912 & 0 & $3.51 E-05$ & 095477 & 0 & $1.95 \mathrm{E}-05$ \\
\hline Q08211 & 1 & 0.999762 & P55010 & 0 & $3.43 E-05$ & Q7RTR2 & 1 & 0.997402 \\
\hline Q8WZA2 & 1 & 0.961994 & P54274 & 1 & 0.999972 & Q9UIK4 & 1 & 0.999918 \\
\hline Q14164 & 0 & 0.000222 & Q9Y239 & 0 & $6.00 \mathrm{E}-05$ & Q5VZM2 & 1 & 0.501929 \\
\hline Q15554 & 1 & 0.999988 & O43237 & 1 & 0.934796 & Q13418 & 0 & $2.04 \mathrm{E}-05$ \\
\hline 095803 & 0 & 4.54E-05 & Q9NQL2 & 1 & 0.999586 & Q8WWZ7 & 0 & $1.86 \mathrm{E}-05$ \\
\hline Q8N8A6 & 1 & 0.999969 & Q00978 & 1 & 0.999994 & Q9HC98 & 1 & 0.999889 \\
\hline Q92730 & 1 & 0.999408 & Q8IZY2 & 0 & 8.83E-05 & Q96C10 & 1 & 0.939997 \\
\hline O14920 & 1 & 0.960552 & Q14934 & 1 & 0.999899 & Q13464 & 0 & 0.000489 \\
\hline Q8WWZ4 & 0 & $5.81 \mathrm{E}-05$ & Q9NZQ0 & 1 & 0.99986 & P20839 & 1 & 0.999961 \\
\hline 000712 & 1 & 0.99999 & P35398 & 1 & 0.99999 & Q86UK0 & 1 & 0.78434 \\
\hline P35659 & 1 & 0.999989 & Q9NWZ3 & 0 & 0.053987 & Q8N4C6 & 0 & 0.000297 \\
\hline P08922 & 0 & $7.01 \mathrm{E}-06$ & P33897 & 0 & $6.48 \mathrm{E}-05$ & Q7Z5P4 & 0 & $3.32 \mathrm{E}-06$ \\
\hline Q08881 & 1 & 0.986495 & Q9C000 & 0 & 0.00102 & Q01974 & 0 & $1.17 \mathrm{E}-05$ \\
\hline P61221 & 0 & $6.47 \mathrm{E}-06$ & P56937 & 1 & 0.999612 & O60674 & 1 & 0.999308 \\
\hline Q86SG6 & 0 & $2.42 \mathrm{E}-05$ & O95398 & 0 & $1.25 \mathrm{E}-05$ & P00519 & 1 & 0.999994 \\
\hline Q9BPW9 & 0 & $1.56 \mathrm{E}-06$ & Q9ULG1 & 1 & 0.999983 & Q8TD19 & 0 & $4.60 \mathrm{E}-05$ \\
\hline Q9Y4G8 & 1 & 0.999992 & O00763 & 0 & 0.000113 & Q9NUU7 & 1 & 0.987564 \\
\hline Q9Y3D8 & 0 & $8.94 \mathrm{E}-06$ & Q12968 & 0 & 0.021484 & Q9HB90 & 0 & $3.60 \mathrm{E}-05$ \\
\hline Q2M218 & 1 & 0.999997 & Q9UMR2 & 1 & 0.976112 & P13861 & 0 & $1.14 \mathrm{E}-05$ \\
\hline Q14938 & 1 & 0.999994 & P10301 & 0 & $2.19 E-05$ & Q9NUT2 & 0 & $3.86 \mathrm{E}-06$ \\
\hline Q08345 & 0 & $6.81 \mathrm{E}-06$ & Q9NYR9 & 0 & $5.68 \mathrm{E}-06$ & P04629 & 0 & $5.34 \mathrm{E}-06$ \\
\hline Q7L523 & 1 & 0.999946 & Q9NRK6 & 0 & $2.40 E-05$ & Q9NR30 & 0 & 0.004318 \\
\hline P48730 & 1 & 0.996475 & Q96RI1 & 1 & 0.999971 & P62070 & 0 & 0.000636 \\
\hline
\end{tabular}




\begin{tabular}{|c|c|c|c|c|c|c|c|c|}
\hline Q09428 & 0 & $5.39 E-05$ & 000571 & 1 & 0.999982 & P49674 & 0 & 0.003382 \\
\hline P78426 & 1 & 0.999956 & Q9BU20 & 0 & $3.14 \mathrm{E}-05$ & P28288 & 0 & $5.25 \mathrm{E}-05$ \\
\hline Q9H0S4 & 0 & $2.99 \mathrm{E}-05$ & P08069 & 0 & 0.000168 & Q9UBE8 & 1 & 0.883397 \\
\hline Q9Y230 & 1 & 0.926172 & Q9UG63 & 1 & 0.999866 & Q9NQI0 & 1 & 0.997146 \\
\hline P51617 & 1 & 0.999924 & P29475 & 0 & 0.043807 & 000442 & 0 & $1.42 \mathrm{E}-06$ \\
\hline Q5FVE4 & 0 & 0.000403 & Q9BQ39 & 1 & 0.92183 & P78368 & 0 & 0.000302 \\
\hline P46459 & 0 & $2.08 \mathrm{E}-06$ & P19793 & 1 & 0.999997 & Q8N139 & 0 & 0.00021 \\
\hline P26196 & 1 & 0.999948 & Q9Y6M4 & 0 & $5.30 \mathrm{E}-05$ & Q8TB37 & 0 & $4.89 \mathrm{E}-06$ \\
\hline Q5K4L6 & 0 & 0.004416 & 094911 & 0 & $1.00 \mathrm{E}-05$ & P09417 & 0 & 0.000556 \\
\hline Q6PHW0 & 1 & 0.996679 & Q5SY16 & 1 & 0.999711 & Q6T310 & 0 & $8.48 \mathrm{E}-05$ \\
\hline Q8IUA7 & 0 & $1.56 \mathrm{E}-05$ & Q6UX07 & 1 & 0.999534 & Q9HCPO & 1 & 0.998032 \\
\hline P35228 & 1 & 0.999666 & Q96HN2 & 1 & 0.980062 & Q9UBJ2 & 0 & 0.153629 \\
\hline Q7L2E3 & 1 & 0.999858 & P12532 & 0 & 0.034041 & 096028 & 1 & 0.999994 \\
\hline Q9NR31 & 0 & $3.67 \mathrm{E}-06$ & Q9UNQ0 & 0 & $2.92 \mathrm{E}-06$ & Q9H2U1 & 1 & 0.999946 \\
\hline P46013 & 1 & 0.999871 & P20393 & 1 & 0.999997 & Q9NZ71 & 1 & 0.999982 \\
\hline Q9H172 & 0 & 4.32E-06 & Q9UPY3 & 0 & 0.12884 & P23458 & 1 & 0.999848 \\
\hline Q9NTK5 & 0 & 4.17E-05 & Q9UBT2 & 0 & $8.49 \mathrm{E}-05$ & Q9H222 & 0 & 0.001491 \\
\hline Q9Y5R6 & 1 & 0.999981 & P52333 & 0 & 0.000537 & Q9Y5Y2 & 0 & $3.06 \mathrm{E}-05$ \\
\hline Q9Y3Z3 & 1 & 0.99076 & Q96GR2 & 0 & 4.51E-05 & Q8NBQ5 & 0 & 7.99E-06 \\
\hline P17612 & 0 & 4.26E-05 & Q13415 & 1 & 0.999984 & Q9Y6B6 & 0 & $1.94 \mathrm{E}-06$ \\
\hline Q9BZC7 & 0 & 0.00011 & O43143 & 1 & 0.999895 & Q13557 & 0 & 8.08E-05 \\
\hline Q9H244 & 0 & $3.20 \mathrm{E}-06$ & Q9UPW6 & 1 & 0.999987 & O75027 & 0 & $1.67 \mathrm{E}-06$ \\
\hline 060231 & 1 & 0.999791 & Q9ULD8 & 1 & 0.998276 & O60285 & 1 & 0.999994 \\
\hline Q9NVA2 & 1 & 0.986201 & 095342 & 0 & $8.52 \mathrm{E}-05$ & P55265 & 1 & 0.999979 \\
\hline O60341 & 1 & 0.999983 & O60313 & 1 & 0.998609 & Q9Y265 & 0 & $1.84 \mathrm{E}-05$ \\
\hline Q8NE71 & 1 & 0.993143 & O60479 & 1 & 0.999996 & Q9Y496 & 1 & 0.998659 \\
\hline Q96RQ9 & 0 & 0.000212 & P57772 & 1 & 0.999386 & Q9NUB1 & 0 & $1.62 \mathrm{E}-06$ \\
\hline P49366 & 0 & $8.61 \mathrm{E}-06$ & P33176 & 1 & 0.999986 & O15294 & 0 & $4.29 E-06$ \\
\hline Q9UHD8 & 1 & 0.999803 & P01011 & 0 & 0.000283 & O43812 & 1 & 0.999992 \\
\hline P31323 & 0 & $3.23 \mathrm{E}-05$ & Q13177 & 0 & 0.000237 & Q9Y6X0 & 1 & 0.999993 \\
\hline Q07912 & 0 & 0.001115 & Q8TE73 & 1 & 0.904735 & Q9NYSO & 0 & $2.58 \mathrm{E}-06$ \\
\hline O75914 & 1 & 0.998539 & Q7Z333 & 1 & 0.999986 & Q9NR19 & 0 & 7.46E-06 \\
\hline Q9C0G6 & 1 & 0.984538 & Q8N752 & 0 & 0.000353 & Q9NQU5 & 1 & 0.999423 \\
\hline Q01826 & 1 & 0.999987 & Q9H2P0 & 1 & 0.999994 & Q9NYC9 & 0 & 0.000227 \\
\hline P48729 & 0 & 0.025907 & 095747 & 1 & 0.998351 & Q53H47 & 1 & 0.99999 \\
\hline Q96CM8 & 0 & $1.55 E-06$ & 095057 & 0 & 0.004414 & Q9BZL6 & 1 & 0.999858 \\
\hline Q9P286 & 1 & 0.999979 & Q9NTG7 & 1 & 0.980596 & Q96P47 & 1 & 0.999987 \\
\hline P50570 & 1 & 0.86177 & Q04759 & 0 & 0.001693 & Q5VST9 & 1 & 0.99968 \\
\hline Q9Y6E7 & 1 & 0.66017 & Q99758 & 0 & 1.36E-05 & 000429 & 0 & 0.002462 \\
\hline P10644 & 1 & 0.589151 & Q9UGN5 & 1 & 0.999965 & Q8N6T7 & 0 & 0.498253 \\
\hline Q86UQ4 & 0 & 0.000111 & Q9Y295 & 0 & $3.47 \mathrm{E}-05$ & Q9UQM7 & 0 & $7.29 E-06$ \\
\hline Q86U86 & 1 & 0.999989 & Q15477 & 1 & 0.999962 & Q2M3G0 & 0 & $6.45 \mathrm{E}-06$ \\
\hline
\end{tabular}




\begin{tabular}{|c|c|c|c|c|c|c|c|c|}
\hline Q6IANO & 1 & 0.997948 & Q15058 & 1 & 0.999691 & Q15645 & 1 & 0.999083 \\
\hline Q7Z7L1 & 0 & 0.007395 & Q9NP58 & 0 & $1.48 \mathrm{E}-06$ & Q01094 & 1 & 0.999979 \\
\hline Q12756 & 1 & 0.697 & Q96RG2 & 0 & 0.000814 & Q15019 & 0 & 0.001216 \\
\hline Q9NP78 & 0 & $1.23 \mathrm{E}-06$ & Q15329 & 1 & 0.999989 & O60333 & 1 & 0.999883 \\
\hline P12004 & 0 & 0.000101 & 000141 & 0 & $2.93 E-05$ & 060706 & 0 & $3.36 \mathrm{E}-06$ \\
\hline 075461 & 1 & 0.999967 & Q03164 & 1 & 0.999987 & Q13153 & 1 & 0.999984 \\
\hline Q8NDV3 & 1 & 0.999953 & 014678 & 0 & $3.53 \mathrm{E}-06$ & Q96AV8 & 1 & 0.999966 \\
\hline Q15139 & 0 & 0.023403 & Q58A45 & 1 & 0.999943 & P28370 & 1 & 0.999987 \\
\hline P45844 & 1 & 0.769148 & P19525 & 1 & 0.999931 & Q02156 & 0 & $5.21 \mathrm{E}-05$ \\
\hline O43252 & 0 & $4.50 \mathrm{E}-06$ & Q9H0K1 & 1 & 0.999971 & P42684 & 1 & 0.999985 \\
\hline Q9P2K8 & 1 & 0.99921 & Q15418 & 0 & 0.006439 & P09874 & 1 & 0.999978 \\
\hline Q8IXJ6 & 0 & 0.053297 & Q4G176 & 0 & $5.62 \mathrm{E}-06$ & A0AVK6 & 1 & 0.999989 \\
\hline Q9H1H9 & 1 & 0.999952 & P40424 & 1 & 0.999997 & Q8N196 & 1 & 0.999934 \\
\hline 060503 & 0 & $3.32 \mathrm{E}-05$ & O60869 & 0 & $3.52 \mathrm{E}-06$ & Q9NQT8 & 1 & 0.995012 \\
\hline O15530 & 0 & 0.000523 & 060264 & 1 & 0.999979 & Q9BRQ8 & 0 & $1.93 \mathrm{E}-05$ \\
\hline P68104 & 0 & 4.32E-06 & Q96L93 & 1 & 0.995907 & P40425 & 1 & 0.999979 \\
\hline Q9H4L7 & 1 & 0.999942 & O60218 & 1 & 0.999951 & 000418 & 0 & 0.001328 \\
\hline Q96Q89 & 1 & 0.999976 & O43933 & 1 & 0.999922 & Q9NXA8 & 0 & 0.118652 \\
\hline Q04828 & 1 & 0.999722 & Q13627 & 1 & 0.999996 & Q9ULI4 & 1 & 0.999989 \\
\hline P51160 & 0 & 0.000115 & Q14683 & 1 & 0.999893 & Q7Z591 & 1 & 0.999991 \\
\hline Q9UBX2 & 1 & 0.999989 & P52732 & 1 & 0.999989 & Q9NTI5 & 1 & 0.999942 \\
\hline Q9UQE7 & 1 & 0.999133 & Q8NI60 & 0 & 0.000319 & 000148 & 1 & 0.995095 \\
\hline Q02241 & 1 & 0.999897 & Q13608 & 1 & 0.99957 & P41225 & 1 & 0.999962 \\
\hline O43306 & 0 & $5.07 \mathrm{E}-06$ & Q9P225 & 0 & 0.000808 & Q05655 & 0 & 0.000203 \\
\hline Q9H792 & 1 & 0.999993 & O43175 & 0 & 0.244253 & P17516 & 1 & 0.999948 \\
\hline Q92630 & 1 & 0.954605 & P41743 & 1 & 0.999973 & Q96BD5 & 1 & 0.999994 \\
\hline Q9Y2K2 & 1 & 0.999987 & Q5T2L2 & 0 & $2.76 \mathrm{E}-06$ & Q16254 & 1 & 0.999969 \\
\hline P14618 & 0 & $1.48 \mathrm{E}-05$ & Q9BXM7 & 0 & $3.78 \mathrm{E}-05$ & Q9NRC8 & 1 & 0.999992 \\
\hline P31749 & 1 & 0.991352 & Q13838 & 1 & 0.500772 & Q8NCM2 & 0 & 0.000142 \\
\hline P17858 & 0 & 4.32E-06 & 000570 & 1 & 0.999968 & P31751 & 1 & 0.996169 \\
\hline Q9P2D7 & 0 & 0.001739 & 000139 & 0 & 0.000491 & Q96RR1 & 1 & 0.99991 \\
\hline Q9UBP0 & 1 & 0.996706 & Q9Y243 & 0 & 0.111731 & Q8WXX0 & 0 & 0.000313 \\
\hline Q12840 & 1 & 0.999988 & P09619 & 0 & 0.000299 & P17947 & 1 & 0.99999 \\
\hline P53396 & 0 & $3.67 \mathrm{E}-06$ & Q14204 & 0 & 0.000165 & O60282 & 1 & 0.999985 \\
\hline P11309 & 0 & 4.22E-06 & Q9UIU6 & 1 & 0.999994 & P36896 & 0 & 3.43E-06 \\
\hline Q9BQI3 & 1 & 0.999981 & Q2M1P5 & 0 & 0.009263 & Q9BVI0 & 1 & 0.99995 \\
\hline P51532 & 1 & 0.999668 & Q08462 & 0 & 3.17E-06 & Q9UQ16 & 1 & 0.983127 \\
\hline P04183 & 0 & 1.17E-06 & P48736 & 1 & 0.999779 & Q969G3 & 1 & 0.999953 \\
\hline 060266 & 0 & 3.10E-05 & 000716 & 1 & 0.999995 & Q8NEZ4 & 1 & 0.999996 \\
\hline Q15126 & 0 & 0.000125 & Q96EB6 & 1 & 0.999871 & Q9UPQ3 & 1 & 0.999975 \\
\hline Q9NZN3 & 1 & 0.739564 & Q13976 & 1 & 0.999584 & Q99453 & 1 & 0.999982 \\
\hline P42285 & 1 & 0.999801 & O94788 & 0 & 0.00015 & P32519 & 1 & 0.999989 \\
\hline
\end{tabular}




\begin{tabular}{|c|c|c|c|c|c|c|c|c|}
\hline Q9P2E2 & 1 & 0.510868 & Q504Y2 & 1 & 0.989171 & P23497 & 1 & 0.999994 \\
\hline Q9H161 & 1 & 0.999996 & Q14209 & 1 & 0.999744 & Q8N4N8 & 1 & 0.999983 \\
\hline Q9WJR5 & 1 & 0.999988 & P38935 & 1 & 0.999951 & Q6IQ32 & 1 & 0.999546 \\
\hline P13639 & 0 & $1.19 \mathrm{E}-05$ & O15066 & 1 & 0.999976 & P63133 & 1 & 0.99999 \\
\hline 095347 & 1 & 0.99961 & P49189 & 0 & $3.04 \mathrm{E}-06$ & Q9NZN4 & 1 & 0.995232 \\
\hline Q9BW19 & 1 & 0.988637 & Q16512 & 1 & 0.999989 & Q9NTJ3 & 1 & 0.999939 \\
\hline Q9UM73 & 0 & $3.07 \mathrm{E}-05$ & P28324 & 1 & 0.999982 & Q9BVG8 & 1 & 0.72686 \\
\hline P53350 & 1 & 0.998686 & Q8IY18 & 1 & 0.998575 & Q99490 & 1 & 0.999986 \\
\hline Q96L91 & 1 & 0.999971 & Q96RR4 & 1 & 0.999992 & Q12837 & 1 & 0.999992 \\
\hline Q96SB8 & 1 & 0.973668 & O14727 & 0 & 0.011741 & Q15375 & 0 & $4.75 \mathrm{E}-05$ \\
\hline Q05513 & 1 & 0.999922 & Q9UQG0 & 1 & 0.999981 & P12931 & 1 & 0.951078 \\
\hline P42330 & 1 & 0.999958 & P29322 & 0 & $7.86 \mathrm{E}-05$ & Q96JN0 & 1 & 0.999989 \\
\hline Q01851 & 1 & 0.999996 & Q08945 & 1 & 0.999189 & P00352 & 0 & $3.93 \mathrm{E}-06$ \\
\hline Q5T890 & 1 & 0.999983 & Q7Z4S6 & 1 & 0.99997 & P10266 & 1 & 0.999978 \\
\hline Q15772 & 1 & 0.999985 & P49419 & 0 & 5.53E-06 & P18074 & 1 & 0.999947 \\
\hline O43896 & 1 & 0.999987 & Q9BXR3 & 1 & 0.99999 & Q9NYA1 & 0 & $1.32 \mathrm{E}-05$ \\
\hline P61204 & 1 & 0.999953 & Q03468 & 1 & 0.999987 & P10721 & 0 & 0.005085 \\
\hline Q07869 & 1 & 0.999953 & Q6ZRS2 & 1 & 0.999959 & P18085 & 0 & 0.422473 \\
\hline P00533 & 0 & 0.000194 & Q9UMN6 & 1 & 0.99999 & P37231 & 0 & 0.002937 \\
\hline P08240 & 1 & 0.985004 & P84085 & 0 & $1.12 \mathrm{E}-05$ & Q04743 & 1 & 0.999994 \\
\hline P05771 & 1 & 0.999736 & P63136 & 1 & 0.999988 & O43704 & 1 & 0.822848 \\
\hline P15121 & 1 & 0.999381 & Q2NKX8 & 1 & 0.999916 & Q15349 & 1 & 0.999833 \\
\hline P63135 & 1 & 0.999978 & Q9P2P6 & 1 & 0.999455 & P84077 & 1 & 0.999232 \\
\hline P07992 & 1 & 0.998766 & P51812 & 0 & 0.00104 & P63132 & 1 & 0.999988 \\
\hline P48431 & 1 & 0.99998 & Q13795 & 0 & $3.77 \mathrm{E}-05$ & O00757 & 1 & 0.52687 \\
\hline 095835 & 1 & 0.999998 & 075360 & 1 & 0.999997 & P35712 & 1 & 0.99999 \\
\hline P56559 & 0 & $1.32 \mathrm{E}-05$ & Q15723 & 1 & 0.999985 & P00338 & 0 & $1.79 \mathrm{E}-05$ \\
\hline Q9NYY3 & 1 & 0.999994 & Q05066 & 1 & 0.999981 & P62330 & 0 & $2.92 \mathrm{E}-06$ \\
\hline P78545 & 1 & 0.999961 & Q9UJU2 & 1 & 0.999994 & Q9H4B4 & 1 & 0.999992 \\
\hline 000338 & 1 & 0.999984 & P40616 & 0 & $6.82 \mathrm{E}-06$ & Q99607 & 1 & 0.999992 \\
\hline P43405 & 0 & 1.15E-05 & P35998 & 0 & 0.000178 & Q8WU08 & 0 & 0.002686 \\
\hline P10398 & 1 & 0.999932 & P21860 & 0 & 0.426325 & P07195 & 0 & 7.64E-06 \\
\hline Q13523 & 1 & 0.999905 & Q9NRA0 & 1 & 0.999772 & Q9Y4B4 & 1 & 0.999996 \\
\hline Q15303 & 0 & $1.50 \mathrm{E}-05$ & Q9UPM6 & 1 & 0.999993 & Q9ULL5 & 1 & 0.999786 \\
\hline Q15208 & 1 & 0.999919 & Q9Y689 & 0 & 0.000892 & Q76MJ5 & 0 & 0.000151 \\
\hline O75449 & 0 & 0.002718 & P54821 & 1 & 0.999994 & Q9Y5M8 & 1 & 0.995301 \\
\hline Q96BM9 & 0 & 0.00015 & P41161 & 1 & 0.999992 & Q9NRM7 & 1 & 0.99999 \\
\hline P62333 & 1 & 0.991166 & Q9Y2H1 & 0 & 0.065693 & Q3SXY8 & 1 & 0.999791 \\
\hline P41212 & 1 & 0.999988 & P53671 & 1 & 0.999956 & P43686 & 0 & $1.09 \mathrm{E}-05$ \\
\hline Q96SB4 & 1 & 0.999996 & Q68CP9 & 1 & 0.999991 & 075460 & 1 & 0.999116 \\
\hline Q6ZMQ8 & 0 & 0.000581 & P62195 & 0 & $1.04 \mathrm{E}-05$ & Q13043 & 1 & 0.999955 \\
\hline Q9NVJ2 & 0 & $1.44 \mathrm{E}-05$ & P11474 & 1 & 0.679108 & Q8IWU2 & 0 & $4.18 \mathrm{E}-05$ \\
\hline
\end{tabular}




\begin{tabular}{|c|c|c|c|c|c|c|c|c|}
\hline Q92620 & 0 & 0.003856 & 094804 & 1 & 0.999632 & Q9NVI7 & 1 & 0.99998 \\
\hline P03372 & 1 & 0.99993 & P36776 & 0 & 0.052683 & P17980 & 0 & 0.014865 \\
\hline Q15831 & 0 & 0.052765 & P36404 & 0 & 0.003517 & P14921 & 1 & 0.999978 \\
\hline Q86WA8 & 1 & 0.999942 & Q92786 & 1 & 0.999984 & Q9Y6E0 & 1 & 0.999884 \\
\hline P49703 & 0 & 0.000128 & P15036 & 1 & 0.99997 & Q38SD2 & 0 & 0.000135 \\
\hline P62191 & 1 & 0.998216 & Q9NRP7 & 0 & 0.112035 & P00966 & 1 & 0.999945 \\
\hline P43268 & 1 & 0.99994 & Q99683 & 1 & 0.994325 & Q13882 & 0 & $3.24 \mathrm{E}-06$ \\
\hline Q9C0K7 & 1 & 0.994359 & Q9NR48 & 1 & 0.999972 & Q8IXL6 & 1 & 0.999928 \\
\hline Q96Q04 & 0 & $6.83 \mathrm{E}-05$ & P31939 & 0 & $8.55 \mathrm{E}-05$ & Q06330 & 1 & 0.999986 \\
\hline O43681 & 0 & $8.80 \mathrm{E}-05$ & P62508 & 1 & 0.999998 & Q5S007 & 0 & 0.0295 \\
\hline Q15257 & 0 & 0.000149 & Q15046 & 1 & 0.997922 & Q8NBU5 & 0 & 0.001157 \\
\hline Q14289 & 1 & 0.998781 & Q9H089 & 0 & 0.000195 & Q96QR8 & 1 & 0.99984 \\
\hline Q9P289 & 1 & 0.996556 & Q96QE3 & 1 & 0.999995 & Q8IYD8 & 1 & 0.999977 \\
\hline Q9Y2U8 & 1 & 0.999993 & O00391 & 0 & 0.045497 & Q9UEW8 & 1 & 0.840721 \\
\hline O43488 & 0 & $3.51 \mathrm{E}-05$ & P49327 & 0 & 4.94E-06 & Q9BXT6 & 1 & 0.995922 \\
\hline 015315 & 1 & 0.999991 & P14868 & 0 & 0.001444 & P36405 & 1 & 0.999982 \\
\hline P16591 & 1 & 0.999471 & Q12851 & 0 & 0.024649 & P51153 & 0 & $3.14 \mathrm{E}-05$ \\
\hline P07814 & 1 & 0.999301 & P40617 & 0 & $1.13 \mathrm{E}-05$ & P02751 & 1 & 0.98987 \\
\hline 060307 & 1 & 0.999709 & P57729 & 1 & 0.997619 & P13984 & 1 & 0.999881 \\
\hline Q9H0F7 & 0 & 4.45E-05 & Q16875 & 1 & 0.999671 & Q9Y6R4 & 1 & 0.989425 \\
\hline P20337 & 0 & 4.38E-06 & Q5JPH6 & 0 & 0.228222 & Q9Y2Q0 & 0 & $2.20 \mathrm{E}-05$ \\
\hline P07332 & 1 & 0.612259 & O95819 & 1 & 0.999969 & P61020 & 0 & $5.74 \mathrm{E}-06$ \\
\hline O75478 & 1 & 0.999993 & Q5T9A4 & 1 & 0.999987 & Q05397 & 1 & 0.997744 \\
\hline Q5TCQ9 & 1 & 0.997215 & Q9NRW1 & 0 & 0.00012 & Q71U36 & 0 & $2.67 \mathrm{E}-06$ \\
\hline Q9ULI0 & 1 & 0.999981 & Q6PIW4 & 1 & 0.999997 & O15021 & 1 & 0.760868 \\
\hline O15067 & 0 & $1.11 \mathrm{E}-05$ & Q7L7X3 & 1 & 0.999981 & Q8WXF7 & 1 & 0.999873 \\
\hline Q9UPW0 & 1 & 0.999992 & P25205 & 1 & 0.998236 & Q9UJV8 & 1 & 0.999994 \\
\hline P04350 & 1 & 0.999124 & Q9P241 & 0 & 0.002098 & Q9H334 & 1 & 0.999952 \\
\hline P33993 & 0 & $3.11 E-05$ & O43502 & 1 & 0.999961 & P68371 & 1 & 0.998995 \\
\hline Q9UIF9 & 1 & 0.999984 & Q9BVA6 & 0 & 0.021211 & P07948 & 1 & 0.999927 \\
\hline Q9H0T7 & 0 & 0.000216 & P07437 & 1 & 0.643341 & Q9NPZ5 & 1 & 0.999752 \\
\hline P55317 & 1 & 0.999992 & Q8IVH8 & 0 & 0.021573 & P61019 & 0 & $1.89 \mathrm{E}-06$ \\
\hline Q9UGU0 & 1 & 0.999994 & Q9BUT1 & 0 & $3.28 \mathrm{E}-06$ & P55318 & 1 & 0.999932 \\
\hline Q96QZ7 & 1 & 0.999994 & Q15771 & 0 & $5.67 \mathrm{E}-06$ & Q9H2K8 & 1 & 0.999985 \\
\hline 094766 & 1 & 0.945005 & Q12948 & 1 & 0.999997 & P49137 & 1 & 0.999504 \\
\hline P63000 & 0 & $2.07 E-06$ & Q03518 & 0 & $1.03 \mathrm{E}-06$ & Q8NFC6 & 1 & 0.999988 \\
\hline Q16676 & 1 & 0.999677 & P27448 & 1 & 0.998955 & P11233 & 0 & 0.001303 \\
\hline Q9UL54 & 1 & 0.999932 & P54132 & 1 & 0.999997 & Q12947 & 1 & 0.999995 \\
\hline Q5TCX8 & 1 & 0.73111 & O75771 & 0 & 0.079169 & Q9UHD2 & 1 & 0.997236 \\
\hline O14965 & 0 & 0.018273 & Q08050 & 1 & 0.999992 & P49736 & 1 & 0.999339 \\
\hline P61026 & 0 & $6.41 \mathrm{E}-06$ & Q14186 & 1 & 0.999986 & Q96GD4 & 1 & 0.999885 \\
\hline Q12778 & 1 & 0.999996 & P33992 & 0 & 0.018568 & O14966 & 0 & 0.000354 \\
\hline
\end{tabular}




\begin{tabular}{|c|c|c|c|c|c|c|c|c|}
\hline Q9GZN2 & 1 & 0.999987 & Q8WY36 & 1 & 0.999979 & P06241 & 1 & 0.690382 \\
\hline P08235 & 1 & 0.999994 & P51151 & 0 & $5.62 \mathrm{E}-05$ & P68366 & 0 & $7.25 \mathrm{E}-05$ \\
\hline Q14692 & 0 & 0.000246 & P11362 & 0 & 0.011763 & Q96JY0 & 1 & 0.999996 \\
\hline 075943 & 0 & 0.014565 & Q9NRH3 & 1 & 0.927319 & P46100 & 1 & 0.999994 \\
\hline P22607 & 0 & 0.000133 & Q16644 & 1 & 0.999902 & Q92878 & 1 & 0.999581 \\
\hline Q15583 & 1 & 0.999974 & Q13705 & 0 & $1.04 \mathrm{E}-05$ & P11413 & 0 & 0.181196 \\
\hline Q14566 & 0 & 0.000333 & Q92698 & 1 & 0.945938 & P10827 & 1 & 0.999997 \\
\hline P51813 & 1 & 0.999592 & Q12952 & 1 & 0.999938 & Q9UJA3 & 0 & 0.004195 \\
\hline P11234 & 0 & 0.000872 & Q03519 & 0 & $2.38 \mathrm{E}-06$ & P33076 & 1 & 0.998557 \\
\hline Q12946 & 1 & 0.999977 & Q9NU22 & 1 & 0.989253 & Q9Y272 & 1 & 0.999923 \\
\hline Q16594 & 1 & 0.999997 & O60566 & 1 & 0.997865 & P55316 & 1 & 0.999996 \\
\hline Q02080 & 1 & 0.999979 & P61106 & 0 & 7.43E-06 & Q587J7 & 1 & 0.999888 \\
\hline Q06187 & 0 & $2.16 \mathrm{E}-05$ & P04150 & 1 & 0.999698 & Q14814 & 1 & 0.999994 \\
\hline P62820 & 0 & $6.87 \mathrm{E}-05$ & P55072 & 0 & 0.000253 & O43683 & 1 & 0.99996 \\
\hline Q9Y261 & 1 & 0.999995 & P29376 & 0 & 0.000811 & Q9ULC3 & 0 & 0.00011 \\
\hline P10828 & 1 & 0.999997 & P15056 & 1 & 0.99991 & Q12950 & 1 & 0.999997 \\
\hline Q9P0L2 & 1 & 0.999945 & P51157 & 1 & 0.998627 & Q8NDG6 & 1 & 0.990309 \\
\hline Q9NSY1 & 1 & 0.999994 & Q12951 & 1 & 0.999996 & Q7KZI7 & 1 & 0.999837 \\
\hline Q13637 & 0 & 0.009192 & P35590 & 0 & 0.001323 & Q8TDC3 & 1 & 0.999961 \\
\hline Q01167 & 1 & 0.99999 & Q96L34 & 1 & 0.999956 & P20336 & 0 & 5.97E-06 \\
\hline Q02763 & 0 & $1.29 \mathrm{E}-05$ & Q8IWQ3 & 1 & 0.999891 & O43524 & 1 & 0.999983 \\
\hline P51608 & 1 & 0.999975 & Q86YS6 & 1 & 0.999946 & Q9UKE5 & 1 & 0.999991 \\
\hline P11586 & 0 & 2.92E-06 & P55040 & 0 & 0.110324 & Q6P0Q8 & 1 & 0.976063 \\
\hline P51148 & 0 & $3.89 E-06$ & Q00059 & 1 & 0.999993 & Q13873 & 1 & 0.998342 \\
\hline P04406 & 0 & $1.59 \mathrm{E}-06$ & P33991 & 1 & 0.999896 & P15918 & 1 & 0.999962 \\
\hline Q14188 & 1 & 0.999971 & Q14781 & 1 & 0.999991 & P32455 & 1 & 0.99907 \\
\hline Q9NXL9 & 1 & 0.999954 & Q61Q22 & 0 & 0.000203 & Q9NWX6 & 1 & 0.989263 \\
\hline P21127 & 1 & 0.999169 & Q9H0R5 & 1 & 0.998352 & Q9Y2H9 & 1 & 0.999949 \\
\hline Q9ULW5 & 0 & $1.37 \mathrm{E}-05$ & 094842 & 1 & 0.999996 & Q9BWU1 & 0 & 0.002961 \\
\hline P32456 & 1 & 0.997686 & P08183 & 0 & $1.47 \mathrm{E}-05$ & P20339 & 0 & $5.26 \mathrm{E}-05$ \\
\hline Q17RP2 & 1 & 0.999969 & P06493 & 0 & 0.000391 & Q49A26 & 1 & 0.999975 \\
\hline Q02078 & 1 & 0.999996 & P20340 & 0 & 0.000188 & 094900 & 1 & 0.999998 \\
\hline Q00534 & 0 & 0.000252 & Q96PP9 & 1 & 0.999944 & 000470 & 1 & 0.999996 \\
\hline Q9H0NO & 1 & 0.999847 & Q96KB5 & 0 & 0.001068 & P50750 & 0 & $9.50 \mathrm{E}-05$ \\
\hline Q96PP8 & 0 & 0.310622 & P50222 & 1 & 0.999984 & P61006 & 0 & $1.53 \mathrm{E}-05$ \\
\hline O14656 & 0 & $5.26 \mathrm{E}-06$ & P60953 & 0 & $1.35 \mathrm{E}-06$ & P38405 & 1 & 0.999839 \\
\hline Q8IXI2 & 1 & 0.998658 & P10114 & 1 & 0.999909 & 043615 & 1 & 0.99987 \\
\hline Q9NYV4 & 1 & 0.992812 & Q03113 & 1 & 0.999974 & P08581 & 1 & 0.9976 \\
\hline Q9Y3L5 & 1 & 0.999435 & Q86UE8 & 1 & 0.998657 & P50613 & 0 & 0.000426 \\
\hline P30679 & 1 & 0.999988 & P40925 & 0 & $2.25 \mathrm{E}-06$ & P62491 & 0 & $3.98 \mathrm{E}-05$ \\
\hline P31314 & 1 & 0.999727 & P07199 & 1 & 0.999979 & P63096 & 0 & $7.55 \mathrm{E}-06$ \\
\hline P21439 & 0 & 4.09E-06 & O00194 & 1 & 0.814398 & O14657 & 0 & 0.000205 \\
\hline
\end{tabular}




\begin{tabular}{|c|c|c|c|c|c|c|c|c|}
\hline Q02224 & 1 & 0.999217 & P11488 & 0 & $1.32 \mathrm{E}-05$ & Q06413 & 1 & 0.999996 \\
\hline Q9H082 & 0 & $1.02 \mathrm{E}-05$ & Q8WZ42 & 1 & 0.964055 & Q99459 & 1 & 0.999974 \\
\hline А8MTJ3 & 0 & $5.01 \mathrm{E}-05$ & O14770 & 1 & 0.999998 & Q96S21 & 1 & 0.998345 \\
\hline Q9Y2W1 & 1 & 0.999835 & 094921 & 1 & 0.999989 & P19086 & 0 & 4.65E-05 \\
\hline Q8IWI9 & 1 & 0.999977 & Q9NP72 & 0 & $2.53 E-05$ & Q8N2E6 & 0 & 0.218778 \\
\hline P24941 & 0 & $1.03 E-05$ & Q9NVN8 & 0 & 0.464423 & Q9H2W2 & 1 & 0.999975 \\
\hline Q9BZG1 & 0 & $1.94 \mathrm{E}-05$ & P11388 & 1 & 0.999996 & Q00535 & 0 & 4.85E-06 \\
\hline Q9HCN4 & 0 & 7.36E-05 & Q8IWA4 & 1 & 0.686439 & Q15286 & 0 & 8.05E-06 \\
\hline Q02880 & 1 & 0.999987 & P49336 & 0 & $1.21 \mathrm{E}-05$ & Q9H9Y4 & 0 & 0.000828 \\
\hline Q8TDZ2 & 0 & 0.001436 & P20338 & 0 & 4.70E-05 & Q5JU69 & 0 & 0.000111 \\
\hline Q96G23 & 1 & 0.997025 & Q8WTQ7 & 0 & 3.93E-05 & P28482 & 1 & 0.999975 \\
\hline P51149 & 1 & 0.998851 & Q6ZSZ6 & 1 & 0.999997 & Q12873 & 1 & 0.999995 \\
\hline P29992 & 1 & 0.93045 & P45983 & 0 & 0.000125 & Q3YEC7 & 1 & 0.999971 \\
\hline Q63HK5 & 1 & 0.999998 & Q14839 & 1 & 0.999991 & 095837 & 0 & 0.000179 \\
\hline Q15759 & 0 & 0.002289 & P15153 & 0 & $2.87 \mathrm{E}-06$ & Q9BXA6 & 0 & 4.65E-05 \\
\hline Q14004 & 1 & 0.999989 & P09471 & 0 & 4.02E-06 & Q96T58 & 1 & 0.999981 \\
\hline Q14088 & 0 & 0.000202 & Q5TCY1 & 1 & 0.999987 & 014647 & 1 & 0.999993 \\
\hline P50148 & 0 & 0.000276 & P53778 & 0 & $3.57 \mathrm{E}-05$ & Q6ZRP7 & 0 & $3.27 \mathrm{E}-05$ \\
\hline Q6IQ55 & 1 & 0.99994 & O14757 & 1 & 0.999735 & P63092 & 0 & 0.362736 \\
\hline Q9HCE1 & 0 & 0.000405 & P57735 & 0 & $2.02 \mathrm{E}-05$ & P33981 & 1 & 0.997961 \\
\hline Q9UQ88 & 1 & 0.999855 & P19087 & 0 & $2.84 \mathrm{E}-05$ & 094851 & 1 & 0.999937 \\
\hline Q13636 & 0 & $8.81 \mathrm{E}-06$ & O95922 & 0 & 0.001091 & 096017 & 1 & 0.999997 \\
\hline P36915 & 1 & 0.999966 & Q7RTP6 & 1 & 0.99997 & Q9NP90 & 0 & 7.67E-06 \\
\hline Q6EMB2 & 1 & 0.999974 & Q96RK0 & 1 & 0.999555 & P11021 & 0 & 0.001265 \\
\hline Q8IXI1 & 0 & $7.97 \mathrm{E}-06$ & Q06609 & 0 & $4.88 \mathrm{E}-06$ & A6PVC2 & 1 & 0.999816 \\
\hline P51793 & 0 & $1.19 \mathrm{E}-06$ & P08754 & 0 & 0.000153 & P53779 & 0 & $2.17 \mathrm{E}-05$ \\
\hline P04049 & 1 & 0.999971 & Q16881 & 0 & $3.58 \mathrm{E}-06$ & P51798 & 0 & $1.94 \mathrm{E}-06$ \\
\hline Q5JWF2 & 1 & 0.999793 & P43246 & 0 & 0.000623 & P62834 & 0 & $6.74 \mathrm{E}-06$ \\
\hline Q86VQ6 & 0 & $3.54 \mathrm{E}-05$ & P49761 & 1 & 0.999998 & Q9BVP2 & 1 & 0.999977 \\
\hline Q92887 & 0 & $2.89 \mathrm{E}-05$ & O14807 & 1 & 0.999958 & Q14679 & 1 & 0.999962 \\
\hline 014646 & 1 & 0.999615 & Q9UHW5 & 0 & 8.73E-06 & O15438 & 0 & $6.85 \mathrm{E}-06$ \\
\hline P01111 & 0 & 0.000145 & P29597 & 0 & 0.000316 & Q8TD26 & 1 & 0.999987 \\
\hline Q969Y2 & 1 & 0.917265 & 095255 & 0 & 0.004013 & P51159 & 0 & $3.70 \mathrm{E}-05$ \\
\hline 075643 & 1 & 0.998914 & Q9HCK8 & 1 & 0.999947 & A4D1E9 & 0 & 0.000487 \\
\hline P27361 & 0 & 0.000907 & Q9UBG7 & 1 & 0.999971 & P17480 & 1 & 0.999989 \\
\hline Q3L8U1 & 1 & 0.999994 & Q14344 & 0 & 0.004563 & P45984 & 0 & 0.000128 \\
\hline P43351 & 1 & 0.999987 & Q9BWV7 & 0 & 0.00102 & P51790 & 0 & $1.01 \mathrm{E}-06$ \\
\hline P04899 & 0 & $4.71 \mathrm{E}-06$ & O15264 & 0 & 0.056712 & P62826 & 0 & 2.97E-06 \\
\hline Q3SXZ7 & 1 & 0.991756 & Q92989 & 0 & 0.000222 & P32298 & 0 & $2.68 \mathrm{E}-05$ \\
\hline Q9NPJ1 & 0 & 0.000116 & P61224 & 0 & 5.99E-06 & P22314 & 1 & 0.994541 \\
\hline 094983 & 1 & 0.999994 & P08631 & 1 & 0.999952 & Q9HBH9 & 0 & 0.005767 \\
\hline Q15907 & 0 & 0.000429 & Q15029 & 0 & 0.00017 & Q8TDI0 & 1 & 0.999995 \\
\hline
\end{tabular}




\begin{tabular}{|c|c|c|c|c|c|c|c|c|}
\hline P62805 & 0 & $2.17 \mathrm{E}-06$ & O14733 & 1 & 0.955448 & P0COE4 & 1 & 0.99991 \\
\hline Q8TBC4 & 0 & $1.17 \mathrm{E}-05$ & Q7L1S5 & 1 & 0.999637 & P48637 & 1 & 0.999856 \\
\hline Q9BT17 & 0 & 1.19E-05 & Q92900 & 0 & 0.000101 & Q8IYT8 & 1 & 0.999557 \\
\hline Q9HAZ1 & 1 & 0.999998 & Q9Y450 & 0 & 0.434943 & 015439 & 0 & $9.27 \mathrm{E}-06$ \\
\hline Q6NUM9 & 0 & $2.92 \mathrm{E}-06$ & P21796 & 0 & 0.002255 & Q9Y259 & 0 & 7.92E-06 \\
\hline Q9BYK8 & 1 & 0.999891 & Q96J65 & 0 & $1.30 \mathrm{E}-05$ & P10276 & 1 & 0.999992 \\
\hline O75385 & 1 & 0.999551 & Q9NRB3 & 1 & 0.999991 & P49773 & 0 & 1.26E-05 \\
\hline P20585 & 1 & 0.999976 & P01112 & 0 & 3.81E-05 & O43314 & 1 & 0.999972 \\
\hline Q7LFX5 & 0 & 0.002061 & Q9P1Z3 & 0 & 0.049036 & Q9Y2G1 & 1 & 0.999979 \\
\hline P01116 & 0 & 0.00028 & P30530 & 0 & 0.000115 & Q9Y6Y1 & 1 & 0.999982 \\
\hline Q9H422 & 1 & 0.999989 & P33527 & 0 & $5.71 \mathrm{E}-06$ & Q8WXH6 & 1 & 0.997792 \\
\hline Q6PHR2 & 1 & 0.998734 & P49759 & 1 & 0.999998 & Q00839 & 1 & 0.999871 \\
\hline O15440 & 0 & $5.70 \mathrm{E}-06$ & Q9UBK7 & 1 & 0.954036 & Q8NBZ7 & 0 & 0.000417 \\
\hline P49760 & 1 & 0.999997 & P20823 & 0 & 0.001348 & Q5T3U5 & 0 & 2.93E-06 \\
\hline Q8TC12 & 0 & $1.28 \mathrm{E}-05$ & Q99986 & 1 & 0.999883 & Q9H078 & 1 & 0.999256 \\
\hline Q14527 & 1 & 0.999962 & P52701 & 1 & 0.999966 & Q8TAl7 & 0 & $3.81 \mathrm{E}-06$ \\
\hline Q9BYP7 & 1 & 0.999984 & Q14028 & 1 & 0.999956 & P17096 & 0 & 0.00055 \\
\hline Q9H4K7 & 1 & 0.999961 & P35250 & 0 & $1.56 \mathrm{E}-06$ & O75351 & 1 & 0.999902 \\
\hline Q96CD2 & 0 & 4.97E-06 & Q9UGU5 & 1 & 0.999985 & O75648 & 0 & $5.54 \mathrm{E}-05$ \\
\hline P48378 & 1 & 0.999953 & Q9H4A3 & 1 & 0.999995 & A5YM72 & 0 & 8.64E-05 \\
\hline Q9Y663 & 1 & 0.924065 & P35580 & 1 & 0.997523 & Q8NBN7 & 0 & 1.33E-05 \\
\hline Q9Y3S1 & 1 & 0.999985 & O43186 & 1 & 0.999994 & Q3SXM5 & 1 & 0.994864 \\
\hline P35749 & 1 & 0.998447 & P40937 & 0 & $6.16 \mathrm{E}-05$ & P35968 & 1 & 0.996741 \\
\hline Q49AN0 & 0 & 0.000535 & P09429 & 1 & 0.999993 & Q86UW6 & 1 & 0.999975 \\
\hline P48380 & 1 & 0.999874 & Q6PFW1 & 1 & 0.9981 & P68400 & 1 & 0.999857 \\
\hline O15347 & 1 & 0.999992 & Bol1T2 & 1 & 0.946302 & Q15382 & 0 & $2.08 \mathrm{E}-05$ \\
\hline O75717 & 1 & 0.999858 & P41240 & 0 & 4.21E-06 & Q8IX15 & 1 & 0.999985 \\
\hline Q9ULV0 & 1 & 0.999977 & P08134 & 0 & 0.489156 & Q9UN37 & 1 & 0.999972 \\
\hline Q13363 & 0 & 0.002392 & Q9BPY8 & 1 & 0.999329 & B2RTY4 & 1 & 0.999959 \\
\hline P57078 & 1 & 0.999923 & Q86Y07 & 1 & 0.999991 & Q8IWT3 & 0 & 0.001316 \\
\hline PODMV9 & 0 & 1.05E-05 & Q9H0A0 & 1 & 0.999576 & P61586 & 0 & 0.279954 \\
\hline Q9NZC7 & 1 & 0.942673 & P19784 & 1 & 0.99994 & P26583 & 1 & 0.999995 \\
\hline P35579 & 1 & 0.999557 & Q99578 & 0 & $6.00 \mathrm{E}-05$ & A3KMH1 & 1 & 0.999957 \\
\hline 014936 & 1 & 0.99857 & P34931 & 0 & 8.91E-06 & 000159 & 1 & 0.997815 \\
\hline Q9UBZ9 & 1 & 0.999986 & O75063 & 0 & 3.67E-06 & O14529 & 1 & 0.999668 \\
\hline P17066 & 0 & $2.99 \mathrm{E}-05$ & O94832 & 1 & 0.999929 & P15927 & 1 & 0.999778 \\
\hline Q14191 & 1 & 0.999987 & Q16526 & 1 & 0.99954 & Q86Z02 & 1 & 0.999993 \\
\hline Q13402 & 1 & 0.999841 & P35251 & 1 & 0.999996 & O75695 & 0 & 8.18E-05 \\
\hline P07333 & 0 & 9.86E-05 & Q6NT76 & 1 & 0.999998 & Q9Y623 & 1 & 0.999618 \\
\hline P35249 & 0 & 0.001441 & Q9H6S0 & 1 & 0.993659 & O14578 & 0 & 0.003192 \\
\hline Q9ULV5 & 1 & 0.999937 & Q9UJ70 & 0 & $2.32 \mathrm{E}-06$ & P22670 & 1 & 0.999964 \\
\hline Q9C0A1 & 1 & 0.999991 & P04839 & 0 & $1.02 \mathrm{E}-06$ & Q9NYD6 & 1 & 0.999247 \\
\hline
\end{tabular}




\begin{tabular}{|c|c|c|c|c|c|c|c|c|}
\hline A7E2Y1 & 0 & 0.037688 & 000212 & 0 & $2.99 \mathrm{E}-06$ & Q15911 & 1 & 0.999976 \\
\hline P39880 & 1 & 0.999984 & P51553 & 0 & $1.11 \mathrm{E}-05$ & Q9UM54 & 1 & 0.999968 \\
\hline Q15669 & 1 & 0.990103 & Q9H4I2 & 1 & 0.999987 & Q92841 & 1 & 0.628048 \\
\hline P11142 & 0 & $6.37 \mathrm{E}-06$ & Q9NX02 & 0 & 0.142945 & P17081 & 0 & 8.87E-07 \\
\hline P43403 & 1 & 0.999967 & Q9UHI6 & 1 & 0.99986 & Q9UPZ9 & 1 & 0.999997 \\
\hline Q9UHQ9 & 0 & $7.60 \mathrm{E}-05$ & Q7L0Q8 & 0 & $2.92 \mathrm{E}-05$ & Q9Y6X8 & 1 & 0.999994 \\
\hline Q5T1V6 & 0 & $5.07 \mathrm{E}-06$ & Q2VIR3 & 0 & 8.37E-05 & 094916 & 1 & 0.999996 \\
\hline 094762 & 1 & 0.999405 & Q5FWF4 & 1 & 0.999966 & Q9Y6G9 & 0 & 0.002581 \\
\hline P41091 & 0 & $1.24 \mathrm{E}-05$ & P08651 & 1 & 0.99867 & Q96D21 & 1 & 0.999931 \\
\hline Q8IYH5 & 1 & 0.999988 & O15075 & 1 & 0.999821 & Q03933 & 1 & 0.999972 \\
\hline P20591 & 1 & 0.999962 & Q13546 & 1 & 0.999977 & Q96TA2 & 0 & 0.0003 \\
\hline Q86XP3 & 1 & 0.994047 & P54652 & 0 & $1.33 \mathrm{E}-05$ & P20592 & 1 & 0.997981 \\
\hline 094844 & 0 & 0.00413 & P37275 & 0 & 0.409687 & Q9NXZ2 & 1 & 0.999884 \\
\hline 060841 & 0 & $3.95 \mathrm{E}-05$ & Q9Y2K3 & 1 & 0.953111 & P62745 & 0 & 8.09E-06 \\
\hline 060315 & 1 & 0.999995 & Q7L014 & 0 & 4.10E-05 & P0DMV8 & 0 & $1.05 \mathrm{E}-05$ \\
\hline Q8WX94 & 1 & 0.998773 & P84095 & 0 & $1.52 \mathrm{E}-06$ & Q9UKY1 & 1 & 0.999987 \\
\hline Q8NHQ9 & 1 & 0.999721 & P31276 & 0 & 0.000269 & Q8IVL1 & 1 & 0.995634 \\
\hline Q9BYZ6 & 0 & 0.000724 & Q86UP3 & 1 & 0.999987 & Q9NY93 & 1 & 0.99999 \\
\hline P12268 & 0 & $3.39 E-05$ & Q9UHB4 & 0 & 0.001302 & O43353 & 1 & 0.999948 \\
\hline O43167 & 1 & 0.999997 & O95786 & 1 & 0.999913 & P09629 & 1 & 0.999993 \\
\hline O95644 & 1 & 0.99999 & Q9ULI2 & 1 & 0.999535 & & & \\
\hline \multicolumn{9}{|l|}{ A.thaliana } \\
\hline pro_id & pred_label & proba & pro_id & pred_label & proba & pro_id & pred_label & proba \\
\hline Q96524 & 1 & 0.999945 & F4KF14 & 1 & 0.989007 & Q94KL5 & 1 & 0.999997 \\
\hline P55826 & 0 & $3.39 E-05$ & Q9FLW0 & 0 & $6.64 \mathrm{E}-05$ & P18064 & 1 & 0.99978 \\
\hline Q9SAJ6 & 0 & 0.068113 & Q9FXG8 & 1 & 0.999995 & Q9C5U3 & 0 & 0.000141 \\
\hline Q9ASS4 & 0 & $1.54 \mathrm{E}-05$ & Q8S8P5 & 1 & 0.999996 & Q8VY13 & 0 & 4.68E-06 \\
\hline Q6EVK6 & 1 & 0.999995 & Q9SZD4 & 0 & 0.012761 & O48788 & 0 & 2.19E-05 \\
\hline Q9XFH4 & 1 & 0.999986 & Q9FY99 & 1 & 0.999852 & Q8S897 & 1 & 0.999995 \\
\hline Q9SEI3 & 1 & 0.965487 & COLGG8 & 0 & $1.38 \mathrm{E}-05$ & Q43125 & 0 & 2.27E-06 \\
\hline Q9SEE5 & 0 & 0.000193 & Q9SנJ3 & 1 & 0.999992 & 004019 & 0 & $1.98 \mathrm{E}-05$ \\
\hline Q0WR59 & 0 & 0.04521 & Q9S7C9 & 1 & 0.999976 & P25858 & 1 & 0.999683 \\
\hline F4IVIO & 1 & 0.987264 & Q9LTX3 & 1 & 0.981241 & Q9FMD7 & 0 & $2.98 \mathrm{E}-06$ \\
\hline Q9FFY9 & 1 & 0.999996 & Q1WIQ6 & 0 & $1.30 \mathrm{E}-06$ & Q9FIM9 & 1 & 0.99992 \\
\hline Q9SEI4 & 0 & 8.55E-05 & Q93Y06 & 0 & 0.000467 & Q9SUP6 & 1 & 0.999956 \\
\hline P27900 & 1 & 0.999998 & Q9ZWC8 & 1 & 0.839059 & Q9MAK9 & 1 & 0.999441 \\
\hline Q9LRP3 & 0 & 0.032099 & Q9SGY1 & 0 & $2.59 \mathrm{E}-06$ & O49506 & 1 & 0.986406 \\
\hline Q9ZPS9 & 1 & 0.7268 & Q9SL67 & 0 & 0.005425 & Q9M8T0 & 0 & $1.56 \mathrm{E}-05$ \\
\hline Q9FWX7 & 0 & 2.43E-05 & Q9SIV3 & 1 & 0.999993 & Q9LZF6 & 0 & 4.06E-05 \\
\hline Q9SSB5 & 0 & $2.29 \mathrm{E}-06$ & Q8VZG8 & 1 & 0.639545 & Q9C8H0 & 0 & $2.51 \mathrm{E}-06$ \\
\hline
\end{tabular}




\begin{tabular}{|c|c|c|c|c|c|c|c|c|}
\hline Q93VR3 & 0 & $2.56 \mathrm{E}-05$ & P54609 & 0 & $5.48 \mathrm{E}-05$ & Q94BQ2 & 0 & 0.000226 \\
\hline Q9FM85 & 1 & 0.999839 & Q9C8J8 & 0 & 0.000169 & Q9LYU4 & 0 & 7.37E-05 \\
\hline Q38870 & 0 & 0.039504 & Q8H1G6 & 0 & 0.090135 & Q9LVN2 & 0 & 0.000467 \\
\hline Q9LHD1 & 0 & $1.63 \mathrm{E}-05$ & Q9LRR9 & 1 & 0.999779 & Q6NLQ6 & 0 & $3.39 \mathrm{E}-05$ \\
\hline Q9SEI2 & 0 & $7.34 \mathrm{E}-05$ & Q9SB61 & 1 & 0.999987 & Q8RWI9 & 1 & 0.986561 \\
\hline Q9LRS0 & 1 & 0.999475 & P92948 & 1 & 0.999986 & Q9SY73 & 0 & 4.30E-05 \\
\hline Q9ZPW7 & 1 & 0.999933 & Q9c9W0 & 0 & 0.102306 & Q9LSV0 & 0 & $5.69 \mathrm{E}-06$ \\
\hline Q06850 & 1 & 0.658048 & Q9SSB4 & 0 & $2.65 \mathrm{E}-06$ & Q9LXG0 & 1 & 0.999985 \\
\hline Q9M2V5 & 0 & 0.006638 & P93031 & 0 & 0.016623 & Q42438 & 0 & $5.53 \mathrm{E}-06$ \\
\hline Q9STF2 & 0 & 0.000261 & Q9FMY7 & 1 & 0.999956 & Q9LJX0 & 0 & $3.98 \mathrm{E}-06$ \\
\hline Q9XIP7 & 0 & $6.31 \mathrm{E}-06$ & Q9ZSA4 & 0 & $6.45 \mathrm{E}-06$ & B9DFG5 & 1 & 0.986668 \\
\hline Q9FKP8 & 1 & 0.999984 & Q84M24 & 0 & $2.61 \mathrm{E}-05$ & Q02283 & 1 & 0.999994 \\
\hline Q9SCN8 & 0 & 0.000197 & Q9ZU40 & 0 & 0.00051 & Q9SVL0 & 1 & 0.99998 \\
\hline Q9C8G9 & 0 & $5.23 \mathrm{E}-06$ & Q94A76 & 0 & $3.32 \mathrm{E}-05$ & P24100 & 0 & 0.000103 \\
\hline Q9SSA8 & 1 & 0.999997 & Q9SEZ1 & 1 & 0.999998 & Q9LZ98 & 0 & 0.005791 \\
\hline Q05466 & 1 & 0.999997 & O80345 & 1 & 0.997427 & Q96529 & 0 & $9.31 \mathrm{E}-05$ \\
\hline Q9LQW3 & 1 & 0.999994 & Q9MAG3 & 1 & 0.698412 & Q0WQG8 & 0 & $5.31 \mathrm{E}-06$ \\
\hline A8R7E6 & 0 & $3.61 \mathrm{E}-06$ & P32746 & 0 & 7.35E-06 & O64722 & 1 & 0.999995 \\
\hline Q84TH5 & 0 & $6.05 E-06$ & Q6STH5 & 0 & $3.34 \mathrm{E}-06$ & Q9ZV56 & 1 & 0.999685 \\
\hline Q9SMQ6 & 0 & $1.37 \mathrm{E}-05$ & Q9FRL5 & 1 & 0.999995 & Q9FIB4 & 1 & 0.99959 \\
\hline Q9ZVW2 & 1 & 0.999962 & O80842 & 1 & 0.999042 & Q9ZRE2 & 0 & 0.000148 \\
\hline Q9FIW9 & 1 & 0.999997 & Q8GZ52 & 1 & 0.998628 & P46604 & 1 & 0.999998 \\
\hline Q8RWC9 & 1 & 0.921751 & Q9CB01 & 0 & $7.29 \mathrm{E}-05$ & Q9FKJ9 & 1 & 0.999519 \\
\hline Q9ZUT8 & 0 & 0.000159 & B9DFX7 & 0 & $7.26 \mathrm{E}-05$ & Q9XIW0 & 0 & 0.183558 \\
\hline Q38902 & 0 & $1.73 \mathrm{E}-06$ & Q9M9S0 & 1 & 0.999992 & Q7PC85 & 0 & $4.25 \mathrm{E}-05$ \\
\hline Q9SZC9 & 0 & 0.012376 & Q9LZW4 & 0 & $3.42 \mathrm{E}-06$ & Q8H156 & 0 & 0.000399 \\
\hline Q9LHF0 & 1 & 0.999996 & Q9LK64 & 0 & $2.80 \mathrm{E}-06$ & O49596 & 1 & 0.999968 \\
\hline Q94C40 & 1 & 0.991912 & Q9LUM4 & 1 & 0.999997 & Q949M9 & 0 & 0.18756 \\
\hline Q9ZUU9 & 0 & $7.08 \mathrm{E}-05$ & Q9LX66 & 0 & $3.45 \mathrm{E}-05$ & Q94CG0 & 0 & 1.15E-05 \\
\hline Q9LM15 & 1 & 0.999977 & B3H5S5 & 0 & 4.17E-05 & Q9M9E1 & 0 & $3.20 \mathrm{E}-06$ \\
\hline P93047 & 1 & 0.999951 & Q93VD3 & 1 & 0.886023 & O23657 & 1 & 0.885452 \\
\hline Q9LPD0 & 0 & 0.322671 & Q9SW08 & 0 & 0.003118 & Q42344 & 1 & 0.999948 \\
\hline Q84VQ3 & 1 & 0.99963 & P28188 & 0 & 2.31E-05 & Q9T074 & 0 & 0.030353 \\
\hline Q9SYI3 & 0 & $3.64 \mathrm{E}-05$ & Q9C9W5 & 0 & $8.98 \mathrm{E}-06$ & F4JY24 & 0 & 0.00516 \\
\hline P31582 & 0 & $6.22 \mathrm{E}-06$ & Q9ZW35 & 1 & 0.99996 & Q9SIT6 & 0 & 0.218228 \\
\hline P51818 & 0 & 0.013414 & QOPCS3 & 1 & 0.999993 & O80501 & 0 & 1.13E-05 \\
\hline Q9SNB4 & 1 & 0.999984 & Q8VZZ4 & 0 & $1.40 \mathrm{E}-05$ & Q9LTT3 & 0 & 0.00028 \\
\hline Q9SN43 & 0 & 0.000132 & Q8W3M3 & 0 & 0.129536 & Q9FE20 & 0 & $3.87 \mathrm{E}-05$ \\
\hline Q9STT5 & 0 & $1.90 \mathrm{E}-05$ & O49597 & 1 & 0.999987 & P25859 & 1 & 0.972792 \\
\hline Q9FJN8 & 0 & 0.004187 & Q9FNP1 & 1 & 0.999958 & Q9ZU35 & 1 & 0.945463 \\
\hline O03986 & 0 & 0.027324 & P16127 & 0 & 4.85E-06 & Q9SIP0 & 1 & 0.999987 \\
\hline Q8RY16 & 0 & 0.094567 & Q9FLX5 & 0 & 0.002251 & F4JFN3 & 1 & 0.999976 \\
\hline
\end{tabular}




\begin{tabular}{|c|c|c|c|c|c|c|c|c|}
\hline Q9SIW2 & 1 & 0.999935 & P92963 & 0 & $1.97 \mathrm{E}-06$ & Q9SSL9 & 0 & $4.40 \mathrm{E}-05$ \\
\hline Q9FLT5 & 0 & $5.97 \mathrm{E}-06$ & Q9LVW2 & 1 & 0.999998 & O22932 & 0 & 0.004797 \\
\hline Q38937 & 0 & $5.73 \mathrm{E}-06$ & Q9LV48 & 1 & 0.999944 & Q9M1C7 & 0 & 0.047053 \\
\hline O49595 & 1 & 0.999898 & Q5XF33 & 0 & $1.45 \mathrm{E}-06$ & Q9SN68 & 0 & $2.34 \mathrm{E}-06$ \\
\hline O49562 & 1 & 0.998684 & Q01593 & 1 & 0.999997 & Q9SGS2 & 1 & 0.999986 \\
\hline Q9FI56 & 0 & 4.13E-05 & P41916 & 0 & 8.43E-05 & Q9MAC9 & 0 & $1.14 \mathrm{E}-05$ \\
\hline Q9SZE1 & 0 & $2.52 \mathrm{E}-05$ & Q43386 & 0 & 0.129153 & Q9SUL7 & 1 & 0.99991 \\
\hline Q7G1L2 & 1 & 0.999977 & Q8W1X2 & 1 & 0.997588 & Q9FKF2 & 0 & $1.69 \mathrm{E}-06$ \\
\hline P27323 & 0 & 0.008145 & Q9C562 & 1 & 0.999988 & Q9SW63 & 1 & 0.999934 \\
\hline Q9FFW5 & 1 & 0.86066 & Q9C8H1 & 0 & 4.01E-06 & Q42525 & 0 & $1.73 \mathrm{E}-05$ \\
\hline Q9SEZ7 & 1 & 0.999982 & P28185 & 0 & 0.009038 & Q9M076 & 0 & $1.52 \mathrm{E}-05$ \\
\hline Q8RXN0 & 0 & $2.79 \mathrm{E}-05$ & P41151 & 1 & 0.999995 & Q9LP51 & 0 & 0.000916 \\
\hline Q38922 & 0 & $2.47 \mathrm{E}-06$ & Q7X9V2 & 1 & 0.999983 & Q9C8K2 & 1 & 0.990306 \\
\hline Q8LDF9 & 1 & 0.99997 & O80902 & 1 & 0.997339 & Q6J9S1 & 1 & 0.999995 \\
\hline Q5YGP8 & 1 & 0.999998 & Q9SKX0 & 0 & 1.19E-05 & P55737 & 0 & 0.002409 \\
\hline Q8W1D5 & 1 & 0.999993 & P28187 & 1 & 0.999977 & Q9S775 & 1 & 0.99997 \\
\hline Q7FB56 & 1 & 0.998203 & Q9SIF2 & 1 & 0.999985 & Q8LF80 & 0 & $3.50 \mathrm{E}-05$ \\
\hline P92978 & 0 & $1.98 \mathrm{E}-06$ & O64682 & 1 & 0.999694 & Q9LSJ6 & 0 & $9.51 \mathrm{E}-06$ \\
\hline Q8RWZ3 & 1 & 0.850846 & Q8VYJ7 & 1 & 0.999156 & Q9SMR4 & 0 & $5.00 \mathrm{E}-05$ \\
\hline Q9C6T1 & 0 & $1.37 \mathrm{E}-05$ & O80946 & 1 & 0.999542 & P93834 & 0 & 2.16E-05 \\
\hline Q9ZUL5 & 1 & 0.999992 & Q9SK03 & 1 & 0.999994 & Q8S9J1 & 1 & 0.999923 \\
\hline Q9C8T1 & 1 & 0.999664 & Q8VZG2 & 1 & 0.999982 & Q94BR5 & 1 & 0.999993 \\
\hline P82280 & 1 & 0.99999 & Q5YGP7 & 1 & 0.999997 & Q9LFG8 & 0 & 0.000622 \\
\hline Q9LPS1 & 0 & 0.000136 & Q9LYQ8 & 1 & 0.999961 & Q9SL02 & 1 & 0.992898 \\
\hline P83483 & 0 & $1.54 \mathrm{E}-05$ & Q7XA72 & 0 & $7.86 \mathrm{E}-05$ & Q8LPJ5 & 0 & 0.000605 \\
\hline Q2V452 & 1 & 0.999954 & P28186 & 0 & 3.37E-05 & P48731 & 1 & 0.999994 \\
\hline Q9LSJ2 & 0 & 3.93E-06 & P41376 & 1 & 0.998655 & Q9LEU7 & 1 & 0.99998 \\
\hline Q8LC30 & 1 & 0.999998 & P46668 & 1 & 0.999995 & Q9FUT3 & 0 & $1.24 \mathrm{E}-05$ \\
\hline Q9SA34 & 0 & $1.91 \mathrm{E}-05$ & O65554 & 1 & 0.999834 & Q8H1E4 & 1 & 0.999979 \\
\hline P83484 & 0 & $1.43 \mathrm{E}-05$ & Q9S725 & 0 & $9.37 \mathrm{E}-06$ & Q56XE8 & 0 & 0.097893 \\
\hline Q9MAM1 & 1 & 0.999982 & P42736 & 1 & 0.999995 & 064629 & 1 & 0.999984 \\
\hline Q9SMT7 & 0 & 4.15E-06 & Q9SCT4 & 0 & $8.55 \mathrm{E}-06$ & Q93Z18 & 0 & 0.001656 \\
\hline Q9FJR0 & 0 & 0.000104 & Q9M077 & 1 & 0.999972 & Q9SH69 & 0 & 0.000141 \\
\hline F4J6F6 & 1 & 0.999944 & Q9SXJ7 & 0 & 0.01144 & Q9FME0 & 1 & 0.999981 \\
\hline Q38897 & 1 & 0.999995 & Q9FFR3 & 0 & 0.021174 & P41377 & 1 & 0.999759 \\
\hline P42762 & 1 & 0.999401 & Q38912 & 0 & 3.05E-06 & P46667 & 1 & 0.999994 \\
\hline Q9M2V6 & 0 & 0.001481 & Q9CAI7 & 1 & 0.998594 & Q9STV4 & 0 & 0.000117 \\
\hline Q38919 & 0 & $4.90 \mathrm{E}-06$ & Q8RWU4 & 1 & 0.999962 & Q9LSJ5 & 0 & $9.11 \mathrm{E}-06$ \\
\hline Q9C5S2 & 0 & 0.000116 & O22971 & 0 & 0.000261 & P41917 & 0 & 4.66E-05 \\
\hline Q94F62 & 0 & $6.23 \mathrm{E}-06$ & Q3EDJ0 & 0 & $5.42 \mathrm{E}-05$ & Q9SUG3 & 0 & 0.003254 \\
\hline P92937 & 1 & 0.999441 & P10896 & 0 & 3.55E-05 & Q1PFD1 & 1 & 0.999976 \\
\hline Q9ZR72 & 0 & 0.000221 & O80568 & 1 & 0.999536 & Q9FJ55 & 0 & $5.18 \mathrm{E}-05$ \\
\hline
\end{tabular}




\begin{tabular}{|c|c|c|c|c|c|c|c|c|}
\hline Q9SD82 & 1 & 0.999989 & Q9SW80 & 1 & 0.999997 & Q9M3B9 & 0 & $3.22 \mathrm{E}-05$ \\
\hline Q9FK35 & 0 & 0.000111 & Q9FJ54 & 1 & 0.999922 & Q9FNM7 & 1 & 0.999739 \\
\hline Q9SIW1 & 1 & 0.999996 & Q9M1Q9 & 0 & $8.46 \mathrm{E}-06$ & Q93YN9 & 1 & 0.999966 \\
\hline Q9LDI3 & 1 & 0.999941 & Q93ZG7 & 0 & $1.90 \mathrm{E}-05$ & Q9M2Z1 & 0 & 7.77E-05 \\
\hline Q93YS4 & 0 & 0.000165 & 081893 & 0 & 0.000191 & P42730 & 0 & 0.000132 \\
\hline Q9SQV1 & 1 & 0.999996 & Q9FWS9 & 1 & 0.999991 & Q3E9B8 & 0 & 0.000805 \\
\hline O82514 & 0 & 4.59E-06 & Q9LF37 & 0 & 0.000941 & Q9LH76 & 0 & $5.01 \mathrm{E}-05$ \\
\hline O49545 & 0 & $3.19 E-05$ & Q9LVM1 & 0 & 0.003822 & Q93VJ2 & 1 & 0.737308 \\
\hline Q9FY74 & 1 & 0.999923 & Q9LPG6 & 0 & 0.003585 & Q9SJ56 & 1 & 0.999997 \\
\hline Q9ZUT0 & 1 & 0.998703 & Q8RWY3 & 0 & 0.000319 & Q6NPP4 & 1 & 0.999983 \\
\hline Q8H136 & 1 & 0.9999 & O65685 & 1 & 0.999998 & Q9LFH0 & 1 & 0.999207 \\
\hline Q9SBA5 & 1 & 0.756115 & Q8GSA7 & 1 & 0.999906 & P47924 & 1 & 0.997331 \\
\hline Q9LZM8 & 1 & 0.999993 & Q9M0D0 & 1 & 0.999009 & Q9FKP4 & 1 & 0.999753 \\
\hline Q9LHE4 & 1 & 0.999987 & Q6NLQ7 & 1 & 0.980829 & 065440 & 0 & 0.013244 \\
\hline Q7PC81 & 0 & 0.06815 & Q9LX99 & 1 & 0.912354 & Q8LPI7 & 1 & 0.961636 \\
\hline Q8LA13 & 1 & 0.999971 & O49397 & 0 & 0.000477 & Q9STT8 & 0 & 8.04E-06 \\
\hline P42158 & 1 & 0.999985 & O23463 & 1 & 0.999978 & Q9SYM5 & 1 & 0.633072 \\
\hline P46897 & 1 & 0.999968 & Q7DM58 & 0 & $2.67 \mathrm{E}-06$ & Q9SWG0 & 0 & $1.08 \mathrm{E}-06$ \\
\hline Q9LSP8 & 1 & 0.999988 & Q9CAM7 & 0 & 0.00177 & Q940D0 & 1 & 0.999987 \\
\hline Q8LPT1 & 0 & $1.68 \mathrm{E}-05$ & P48001 & 1 & 0.99998 & Q9FIL7 & 0 & 0.003342 \\
\hline Q9M2F9 & 0 & 0.011021 & P92549 & 0 & 0.000132 & Q9CAF5 & 1 & 0.971603 \\
\hline Q9FPQ8 & 1 & 0.999872 & Q9FYG2 & 1 & 0.99998 & Q9FN89 & 1 & 0.98391 \\
\hline Q9ZPY9 & 0 & 0.00016 & Q9LK62 & 0 & 2.27E-05 & P46640 & 1 & 0.999985 \\
\hline Q8VZJ9 & 1 & 0.999992 & Q9LVI6 & 0 & 0.000267 & Q11207 & 1 & 0.999985 \\
\hline Q8LPK0 & 0 & $7.80 \mathrm{E}-06$ & Q9LDN0 & 0 & 0.000685 & O49564 & 0 & $1.58 \mathrm{E}-05$ \\
\hline Q9FFH1 & 1 & 0.999945 & Q93V99 & 1 & 0.999947 & Q9M0M2 & 0 & $5.46 \mathrm{E}-06$ \\
\hline P46639 & 1 & 0.999997 & Q9LDT0 & 0 & $3.71 \mathrm{E}-06$ & Q8W4J9 & 0 & $3.70 \mathrm{E}-05$ \\
\hline Q9M7Q7 & 1 & 0.999972 & Q8H1R4 & 0 & $1.65 \mathrm{E}-05$ & Q84JS6 & 1 & 0.999969 \\
\hline Q9LDN1 & 1 & 0.937857 & Q42484 & 1 & 0.999935 & Q84WU8 & 0 & $5.10 \mathrm{E}-06$ \\
\hline Q9M3D6 & 0 & 0.004301 & P92958 & 0 & $1.53 \mathrm{E}-05$ & Q9XEC7 & 1 & 0.999934 \\
\hline 064973 & 1 & 0.999619 & Q8VZI8 & 0 & 0.091569 & Q94FB9 & 0 & 0.000153 \\
\hline P48000 & 1 & 0.99999 & Q9CAL2 & 0 & $1.53 \mathrm{E}-05$ & F4HY56 & 1 & 0.998793 \\
\hline Q8L743 & 1 & 0.999995 & Q9XF19 & 0 & 9.59E-06 & P42818 & 1 & 0.999632 \\
\hline Q9FNE1 & 0 & 0.02149 & Q8L840 & 1 & 0.999945 & Q8VYG2 & 0 & $6.65 \mathrm{E}-06$ \\
\hline Q9M0G9 & 0 & 0.002697 & Q38997 & 0 & $5.25 \mathrm{E}-05$ & Q9C5S8 & 1 & 0.999955 \\
\hline Q39214 & 1 & 0.68487 & Q9M092 & 1 & 0.999994 & Q8RY46 & 0 & $7.82 \mathrm{E}-06$ \\
\hline Q8L7Y8 & 0 & 0.020301 & Q9M9Y8 & 0 & 4.40E-06 & Q9M667 & 1 & 0.999989 \\
\hline Q9SJK6 & 0 & $3.59 \mathrm{E}-05$ & Q8LPQ6 & 0 & $3.71 \mathrm{E}-06$ & P48002 & 1 & 0.999905 \\
\hline Q9ZP16 & 0 & 0.001807 & Q9FT70 & 1 & 0.999977 & Q9C5T3 & 1 & 0.999988 \\
\hline Q94A18 & 0 & 7.14E-05 & P43288 & 0 & $1.72 \mathrm{E}-05$ & Q0PW40 & 0 & 0.264263 \\
\hline Q9SJT1 & 1 & 0.999967 & Q93WU9 & 1 & 0.999998 & Q6NLC1 & 0 & $5.91 \mathrm{E}-05$ \\
\hline Q9SJD4 & 0 & $6.08 \mathrm{E}-06$ & Q8L710 & 0 & 0.037492 & Q9LK36 & 0 & $1.24 \mathrm{E}-06$ \\
\hline
\end{tabular}




\begin{tabular}{|c|c|c|c|c|c|c|c|c|}
\hline 004292 & 1 & 0.959542 & Q9FLT4 & 0 & $2.03 E-06$ & Q940B8 & 1 & 0.999914 \\
\hline O65472 & 0 & 4.47E-06 & Q8GYD9 & 1 & 0.999928 & Q9FXD6 & 1 & 0.99997 \\
\hline Q9MAH4 & 1 & 0.999889 & Q39011 & 0 & $3.51 \mathrm{E}-05$ & Q8W4G6 & 0 & 0.111745 \\
\hline Q9SKB2 & 1 & 0.999971 & Q9ZWJ9 & 1 & 0.999994 & Q9FLT8 & 0 & $2.00 \mathrm{E}-06$ \\
\hline Q38933 & 0 & $4.04 \mathrm{E}-05$ & Q8RX80 & 0 & 0.000561 & Q39203 & 0 & $3.41 \mathrm{E}-05$ \\
\hline Q07970 & 0 & 0.195884 & Q9C7F8 & 0 & 0.149475 & Q9CAP8 & 0 & $5.53 \mathrm{E}-06$ \\
\hline Q9LMB9 & 1 & 0.999883 & 004130 & 0 & $3.48 \mathrm{E}-06$ & Q8LAH7 & 0 & 0.000774 \\
\hline Q9LZJ5 & 0 & $6.65 \mathrm{E}-06$ & Q9M9L8 & 0 & 0.00012 & O65479 & 0 & 0.000359 \\
\hline O81905 & 1 & 0.989118 & Q8GYB8 & 1 & 0.988186 & Q9M2V7 & 0 & 0.024723 \\
\hline F4I3V3 & 1 & 0.964043 & Q6NQ87 & 0 & 0.000685 & Q8RWZ5 & 0 & 0.000233 \\
\hline Q9SGW8 & 1 & 0.998624 & Q9LK50 & 1 & 0.999654 & Q00958 & 1 & 0.999962 \\
\hline O64817 & 0 & $1.95 \mathrm{E}-05$ & D8WUA4 & 1 & 0.999963 & Q9FUP0 & 0 & $2.91 \mathrm{E}-05$ \\
\hline Q9FT51 & 0 & $3.02 E-05$ & P93655 & 1 & 0.582115 & O65476 & 0 & $4.53 \mathrm{E}-05$ \\
\hline Q01474 & 0 & 0.001555 & O82533 & 0 & 0.221157 & Q84K47 & 0 & $1.41 \mathrm{E}-05$ \\
\hline Q9M9L7 & 0 & 0.035483 & Q8GWJ7 & 1 & 0.999902 & O82663 & 1 & 0.995518 \\
\hline Q9M0V6 & 1 & 0.999973 & Q8LPJ4 & 0 & $8.90 \mathrm{E}-06$ & F4HQ17 & 1 & 0.999988 \\
\hline Q3E9X6 & 0 & 0.001594 & Q9ZPX5 & 0 & $2.07 \mathrm{E}-05$ & Q42545 & 1 & 0.999912 \\
\hline O81016 & 0 & $1.65 E-05$ & F4KA50 & 1 & 0.999938 & Q9T0J1 & 0 & 0.00241 \\
\hline Q9SYI0 & 1 & 0.999934 & Q9FKW6 & 0 & 0.000343 & Q7PC86 & 0 & $2.52 \mathrm{E}-05$ \\
\hline F4HQ23 & 0 & $4.72 \mathrm{E}-05$ & Q8S9L6 & 0 & 0.000519 & Q9XIC7 & 0 & $5.45 \mathrm{E}-06$ \\
\hline Q9C9L5 & 1 & 0.998331 & Q9XIE2 & 0 & $5.29 \mathrm{E}-06$ & Q9SJF1 & 0 & 0.000632 \\
\hline Q9LDS6 & 1 & 0.62633 & Q9XGX0 & 1 & 0.999997 & Q9S9M5 & 0 & 0.017267 \\
\hline Q7PC84 & 0 & $5.27 \mathrm{E}-05$ & Q9S7U9 & 0 & 0.000149 & Q9LDQ3 & 0 & 4.01E-05 \\
\hline Q8L607 & 0 & 0.002214 & Q9S9M2 & 1 & 0.999898 & Q1PEH6 & 0 & $7.64 \mathrm{E}-06$ \\
\hline Q39008 & 1 & 0.999984 & Q9SYS7 & 1 & 0.764143 & O24629 & 1 & 0.999972 \\
\hline Q8RY17 & 1 & 0.999476 & Q9SYI2 & 0 & $1.30 \mathrm{E}-05$ & Q9SFB6 & 0 & 0.000108 \\
\hline Q8L7G3 & 1 & 0.990934 & P52839 & 0 & 0.005046 & COLGH2 & 0 & $1.09 \mathrm{E}-05$ \\
\hline O80725 & 0 & $7.88 \mathrm{E}-06$ & Q9LFH9 & 0 & $3.85 \mathrm{E}-06$ & 065483 & 0 & 4.64E-06 \\
\hline Q9SKG5 & 0 & 4.84E-06 & O04567 & 0 & 0.11688 & Q9M1H3 & 0 & 0.001953 \\
\hline P0C5E2 & 1 & 0.999966 & Q9LYU7 & 1 & 0.999293 & Q9M1T1 & 0 & 0.005212 \\
\hline Q944A7 & 1 & 0.974559 & Q9STT7 & 0 & 2.93E-06 & F4HQ22 & 0 & $5.85 \mathrm{E}-05$ \\
\hline Q9ASQ5 & 1 & 0.999949 & Q9M8S6 & 0 & 2.95E-05 & Q9FHD7 & 1 & 0.998233 \\
\hline Q9LV93 & 1 & 0.999919 & D7SFH9 & 0 & 0.000267 & Q8GYA4 & 1 & 0.999955 \\
\hline Q9C9C9 & 0 & 0.000157 & Q8RYC8 & 1 & 0.999996 & Q9STT6 & 0 & 8.06E-06 \\
\hline Q94CK9 & 1 & 0.999996 & Q8H199 & 0 & $7.62 \mathrm{E}-06$ & Q9FII7 & 1 & 0.999995 \\
\hline Q9C8N9 & 1 & 0.999956 & Q9FNB5 & 1 & 0.999103 & Q9SHS8 & 1 & 0.99792 \\
\hline O65482 & 1 & 0.999766 & Q8L7L1 & 0 & $7.64 \mathrm{E}-05$ & Q9LP07 & 1 & 0.99989 \\
\hline Q8LGU1 & 0 & 2.15E-06 & Q94A06 & 1 & 0.999741 & O65405 & 0 & 3.45E-06 \\
\hline Q9SYX2 & 1 & 0.999993 & Q9SZX3 & 1 & 0.996012 & P06525 & 0 & 0.000313 \\
\hline Q9FF31 & 1 & 0.999928 & Q9LDM5 & 0 & 0.11659 & Q9FJL0 & 1 & 0.838377 \\
\hline Q9ZPX1 & 1 & 0.999876 & Q9LYS2 & 0 & 0.000101 & F4KA51 & 1 & 0.999968 \\
\hline Q9XEC8 & 0 & 0.001444 & Q9FZ80 & 1 & 0.999704 & 004291 & 1 & 0.999333 \\
\hline
\end{tabular}




\begin{tabular}{|c|c|c|c|c|c|c|c|c|}
\hline Q8LEF6 & 0 & 0.000529 & F4HQ20 & 0 & 0.000358 & Q9SYS3 & 1 & 0.988618 \\
\hline Q39192 & 0 & 0.002257 & Q66GP9 & 0 & $1.57 \mathrm{E}-05$ & Q9FWX8 & 0 & $1.88 \mathrm{E}-05$ \\
\hline Q3ECH2 & 0 & 0.001807 & Q9LZU4 & 1 & 0.99411 & Q39193 & 0 & $9.59 \mathrm{E}-06$ \\
\hline Q9SB48 & 1 & 0.991516 & Q9C7F2 & 0 & 0.00033 & Q9LSR8 & 0 & $1.29 \mathrm{E}-05$ \\
\hline O65468 & 1 & 0.999249 & Q9SD40 & 1 & 0.999997 & Q9SLG0 & 1 & 0.999989 \\
\hline Q9C6W5 & 0 & 0.000365 & Q9M9E0 & 0 & $1.96 \mathrm{E}-05$ & Q9FIU5 & 0 & 4.26E-06 \\
\hline Q9LQZ5 & 1 & 0.999994 & P18616 & 1 & 0.999971 & Q9LSJ8 & 0 & 1.65E-05 \\
\hline Q9C6K9 & 1 & 0.999997 & Q08467 & 1 & 0.999972 & F4KH89 & 1 & 0.999997 \\
\hline Q9M8Z5 & 1 & 0.999948 & Q9FJH6 & 1 & 0.999982 & Q8VYG0 & 0 & 0.029685 \\
\hline Q08466 & 0 & 0.001646 & Q56YN8 & 1 & 0.999858 & Q9C923 & 1 & 0.999616 \\
\hline QOWMLO & 0 & $4.87 \mathrm{E}-06$ & Q9LJD8 & 0 & 0.0011 & O64816 & 1 & 0.999844 \\
\hline Q9LUL4 & 1 & 0.995699 & Q9S7E4 & 0 & $1.66 \mathrm{E}-06$ & Q9FF46 & 0 & $2.57 \mathrm{E}-05$ \\
\hline Q9LV58 & 1 & 0.999935 & P27450 & 0 & $1.53 \mathrm{E}-05$ & Q940H6 & 0 & 0.000229 \\
\hline Q9S9P8 & 1 & 0.999996 & Q9LZB8 & 0 & 0.054104 & Q9SJI8 & 1 & 0.999806 \\
\hline Q9M0X5 & 1 & 0.999969 & P43292 & 0 & 0.000382 & Q9SCZ4 & 1 & 0.998848 \\
\hline Q8LPK2 & 0 & $8.88 \mathrm{E}-06$ & O22042 & 1 & 0.999924 & Q9CAL3 & 0 & 0.024336 \\
\hline Q38874 & 1 & 0.999998 & Q8W493 & 1 & 0.999949 & Q42093 & 0 & $3.90 \mathrm{E}-06$ \\
\hline P93819 & 0 & $1.14 \mathrm{E}-06$ & Q9XEC6 & 1 & 0.998656 & O22558 & 0 & 0.004765 \\
\hline Q9FGM0 & 1 & 0.998002 & Q7PC88 & 0 & $6.41 \mathrm{E}-06$ & Q8RXG3 & 1 & 0.999979 \\
\hline O23081 & 0 & 0.000432 & Q9C9D0 & 1 & 0.996301 & Q9LXJ0 & 1 & 0.998128 \\
\hline Q7PC87 & 0 & 7.97E-06 & Q9FZ36 & 1 & 0.976226 & Q9C5S9 & 1 & 0.999797 \\
\hline Q9M2U4 & 1 & 0.999985 & Q9LN59 & 1 & 0.999776 & Q8H0V6 & 1 & 0.997295 \\
\hline Q9LYG3 & 1 & 0.981193 & O80574 & 0 & $1.74 \mathrm{E}-06$ & Q8RWL6 & 0 & 0.000104 \\
\hline Q9S9M3 & 1 & 0.999918 & Q7PC83 & 0 & 0.007967 & Q9LPD9 & 1 & 0.99995 \\
\hline P84634 & 1 & 0.992711 & F2Y4A3 & 0 & 0.4558 & O22176 & 1 & 0.999998 \\
\hline Q7PC82 & 0 & 0.0019 & Q9FL33 & 0 & 0.342939 & Q93Y16 & 1 & 0.999938 \\
\hline Q8RWZ1 & 0 & 0.184916 & Q9STX0 & 1 & 0.999998 & Q7GB25 & 0 & 2.33E-05 \\
\hline P23686 & 0 & $1.39 \mathrm{E}-06$ & Q3EBC8 & 1 & 0.999976 & Q9C5P1 & 1 & 0.999989 \\
\hline Q9ZNT0 & 1 & 0.999984 & Q9FHF1 & 0 & $2.39 \mathrm{E}-05$ & Q9LXT3 & 1 & 0.999915 \\
\hline Q38953 & 1 & 0.998532 & Q9C8M9 & 1 & 0.79389 & Q0WNY5 & 0 & 0.015065 \\
\hline Q9LHK4 & 0 & 3.13E-06 & QOWVF5 & 1 & 0.999988 & Q8VYV7 & 0 & 0.000104 \\
\hline Q9SI19 & 1 & 0.999993 & Q9XI90 & 1 & 0.999993 & Q9SZR9 & 0 & $6.51 \mathrm{E}-06$ \\
\hline O80786 & 0 & 6.59E-05 & Q9SP32 & 1 & 0.992868 & Q9FXH7 & 1 & 0.999995 \\
\hline O80462 & 0 & $3.39 E-05$ & Q8L7L5 & 1 & 0.534148 & Q9FT73 & 1 & 0.999949 \\
\hline Q9LXW7 & 1 & 0.967415 & P37107 & 0 & $5.78 \mathrm{E}-06$ & O80623 & 1 & 0.996342 \\
\hline A1L4X7 & 1 & 0.999991 & Q9LUT2 & 0 & $1.56 \mathrm{E}-06$ & Q9M5K2 & 0 & $1.36 \mathrm{E}-06$ \\
\hline O65517 & 1 & 0.999998 & C6KIE6 & 1 & 0.999708 & Q9SJG4 & 1 & 0.999984 \\
\hline Q8W3L1 & 0 & 0.000116 & O82497 & 1 & 0.999806 & Q9SJT8 & 1 & 0.999998 \\
\hline COLGN2 & 0 & 4.87E-06 & O22130 & 1 & 0.999996 & F4KAB8 & 1 & 0.945891 \\
\hline Q9FNY3 & 1 & 0.999987 & Q05153 & 1 & 0.999982 & Q0WTB4 & 0 & 0.00508 \\
\hline Q8GXB3 & 1 & 0.999993 & P57106 & 0 & $1.01 \mathrm{E}-06$ & Q8H1F6 & 1 & 0.999992 \\
\hline F4HW65 & 1 & 0.999992 & O23661 & 1 & 0.999979 & Q9LID6 & 0 & $1.00 \mathrm{E}-05$ \\
\hline
\end{tabular}




\begin{tabular}{|c|c|c|c|c|c|c|c|c|}
\hline Q9LKA3 & 0 & $2.86 \mathrm{E}-06$ & Q9SYS6 & 1 & 0.99999 & Q9C5P4 & 1 & 0.999992 \\
\hline P93024 & 1 & 0.999991 & Q8LPN5 & 1 & 0.999997 & Q8LCU7 & 0 & 0.000118 \\
\hline Q8S3C9 & 1 & 0.999745 & F4IHS2 & 1 & 0.999981 & Q9FGV1 & 1 & 0.9788 \\
\hline Q9LTA2 & 1 & 0.999994 & P43299 & 1 & 0.999815 & Q9SE83 & 0 & 0.000128 \\
\hline O23627 & 1 & 0.994382 & Q9SKN5 & 1 & 0.999957 & Q9SR17 & 1 & 0.999996 \\
\hline Q9SJL8 & 0 & $9.06 \mathrm{E}-07$ & P93835 & 1 & 0.999993 & F4JTP5 & 1 & 0.982298 \\
\hline Q9ZPY6 & 1 & 0.998324 & Q8GWQ2 & 1 & 0.999963 & Q9ZP06 & 0 & $1.65 \mathrm{E}-06$ \\
\hline O82132 & 1 & 0.999996 & Q9C5P0 & 1 & 0.999963 & Q84K00 & 1 & 0.999965 \\
\hline Q0PGJ6 & 0 & 0.000141 & Q9MBA2 & 1 & 0.999068 & Q9FHI7 & 0 & 0.008932 \\
\hline Q9LZV4 & 1 & 0.956703 & Q9SKT7 & 1 & 0.999766 & Q9M338 & 0 & $2.73 \mathrm{E}-05$ \\
\hline P17562 & 0 & $2.75 \mathrm{E}-06$ & Q9M5K3 & 0 & $1.79 \mathrm{E}-06$ & Q9M084 & 1 & 0.999963 \\
\hline Q9ZNT1 & 0 & $3.05 E-06$ & Q96329 & 0 & $9.40 \mathrm{E}-05$ & Q39023 & 0 & 0.002705 \\
\hline Q9MOL0 & 1 & 0.999984 & Q9SSK1 & 0 & 0.000132 & Q94BV7 & 1 & 0.99998 \\
\hline Q940I0 & 1 & 0.999995 & Q9C6D2 & 0 & $6.48 \mathrm{E}-05$ & P42697 & 1 & 0.998447 \\
\hline Q9SW96 & 0 & $2.86 \mathrm{E}-06$ & Q6NPS8 & 1 & 0.999869 & O23620 & 1 & 0.99994 \\
\hline O24617 & 1 & 0.999947 & Q9LQ55 & 0 & 0.001361 & Q9ZPI1 & 1 & 0.998642 \\
\hline P33207 & 0 & $1.08 \mathrm{E}-06$ & O49658 & 1 & 0.999997 & Q9SMV7 & 1 & 0.999929 \\
\hline Q8LFT2 & 1 & 0.950208 & P38558 & 1 & 0.999955 & O49213 & 0 & 9.96E-06 \\
\hline Q9FIR1 & 1 & 0.999996 & Q39024 & 0 & 0.000127 & Q9T0J6 & 1 & 0.996818 \\
\hline Q9AT00 & 0 & 0.021488 & Q9LMU0 & 0 & 4.28E-05 & Q9FW44 & 0 & 0.000681 \\
\hline Q39026 & 0 & 0.001216 & Q9FV70 & 1 & 0.999993 & P38557 & 1 & 0.999917 \\
\hline Q9MB58 & 1 & 0.993719 & Q9M9R4 & 1 & 0.999962 & 004716 & 1 & 0.999988 \\
\hline P0DI16 & 0 & 0.00092 & Q9LK35 & 0 & 1.69E-05 & Q8W035 & 0 & 0.339709 \\
\hline Q9SB31 & 1 & 0.999986 & Q9LK95 & 1 & 0.999998 & Q84XF3 & 0 & 0.001574 \\
\hline Q56WH1 & 0 & $1.57 \mathrm{E}-06$ & Q9C5T4 & 1 & 0.999995 & Q4V3E0 & 1 & 0.999967 \\
\hline Q9SZP1 & 1 & 0.999998 & F4HPR5 & 1 & 0.999967 & B9DHQ0 & 0 & $1.57 \mathrm{E}-06$ \\
\hline Q9SB92 & 1 & 0.999993 & O80834 & 1 & 0.994408 & Q9FE25 & 1 & 0.999716 \\
\hline Q05762 & 0 & $6.64 \mathrm{E}-05$ & Q9SLF3 & 1 & 0.999843 & Q9C516 & 1 & 0.999983 \\
\hline Q38898 & 0 & 0.001555 & F4I460 & 1 & 0.999981 & Q8RWL0 & 1 & 0.999962 \\
\hline Q9LUS2 & 1 & 0.998718 & Q9C8M5 & 1 & 0.999985 & Q9SCX5 & 0 & $6.87 \mathrm{E}-06$ \\
\hline F4IRU3 & 1 & 0.999976 & Q9FNY2 & 1 & 0.999997 & O81283 & 1 & 0.998303 \\
\hline Q959M1 & 0 & 0.041316 & Q56YU0 & 0 & $1.36 \mathrm{E}-06$ & Q0WPU1 & 1 & 0.999989 \\
\hline 082133 & 1 & 0.999999 & Q8H0U5 & 0 & 0.020929 & Q9SEI0 & 1 & 0.999998 \\
\hline Q9SZ70 & 1 & 0.999996 & Q9M2K0 & 1 & 0.999987 & Q9FHJ2 & 1 & 0.999969 \\
\hline F4IW47 & 0 & $3.02 \mathrm{E}-06$ & Q9LZV6 & 1 & 0.999993 & Q9LVB0 & 1 & 0.999996 \\
\hline F4K5J1 & 1 & 0.999977 & Q8LF21 & 1 & 0.998987 & Q9FYK0 & 0 & 0.006449 \\
\hline Q9SXB8 & 0 & $1.26 \mathrm{E}-05$ & O49662 & 1 & 0.999995 & F4HXP9 & 1 & 0.999958 \\
\hline Q9LQK0 & 0 & $1.46 \mathrm{E}-05$ & Q8RWV0 & 0 & 2.23E-06 & C0LGJ1 & 0 & 0.000532 \\
\hline Q9C9K7 & 1 & 0.999991 & F4HYV5 & 1 & 0.999998 & Q9CAl1 & 0 & 4.60E-05 \\
\hline O23680 & 1 & 0.999535 & Q9ZQ70 & 1 & 0.999997 & Q9FHM5 & 1 & 0.999996 \\
\hline Q9C8W9 & 1 & 0.999996 & Q9SVA6 & 0 & $6.57 \mathrm{E}-06$ & Q6S5G3 & 1 & 0.999964 \\
\hline Q9LQE8 & 1 & 0.999983 & Q84TF0 & 0 & 0.003307 & Q9LKG8 & 1 & 0.999846 \\
\hline
\end{tabular}




\begin{tabular}{|c|c|c|c|c|c|c|c|c|}
\hline F4IBE4 & 0 & 0.00092 & P43298 & 0 & $7.57 \mathrm{E}-05$ & Q84WU6 & 1 & 0.999974 \\
\hline Q8GXE6 & 0 & $1.52 E-05$ & 004017 & 1 & 0.999991 & Q84WD3 & 1 & 0.999992 \\
\hline Q9LK43 & 0 & $2.98 \mathrm{E}-05$ & Q9LSD6 & 0 & 1.83E-05 & O22812 & 1 & 0.999993 \\
\hline F4HWY6 & 1 & 0.999972 & Q8VZC7 & 1 & 0.999959 & P30182 & 1 & 0.999957 \\
\hline Q9FKT0 & 0 & $6.10 \mathrm{E}-05$ & Q9M2S3 & 1 & 0.999997 & F4JIU4 & 1 & 0.999913 \\
\hline Q8W4H7 & 0 & 4.05E-06 & Q9M2X3 & 1 & 0.999972 & Q84N64 & 1 & 0.99988 \\
\hline Q9LZX7 & 1 & 0.999881 & Q9LKB9 & 1 & 0.999985 & Q8GTY0 & 0 & 4.05E-06 \\
\hline Q9FJW5 & 1 & 0.999989 & Q38851 & 1 & 0.999996 & Q8VYJ2 & 1 & 0.999997 \\
\hline F4I5Q6 & 1 & 0.999983 & Q9ZT91 & 0 & 0.000467 & Q9CA28 & 0 & 0.36771 \\
\hline F4IVR7 & 1 & 0.99999 & O82166 & 1 & 0.999991 & Q9SCL7 & 0 & $4.39 \mathrm{E}-05$ \\
\hline Q9LW09 & 0 & 0.000367 & Q9SIT1 & 0 & 0.000106 & F4JM19 & 1 & 0.99998 \\
\hline Q6DBQ1 & 1 & 0.999988 & Q9FNV8 & 1 & 0.99997 & Q9SSR8 & 1 & 0.994606 \\
\hline Q8VWK4 & 1 & 0.999996 & F4K0A6 & 1 & 0.999959 & Q38998 & 0 & $3.83 \mathrm{E}-06$ \\
\hline Q96276 & 1 & 0.999997 & Q9FNX5 & 1 & 0.999968 & Q6R0E3 & 1 & 0.999991 \\
\hline Q9LD44 & 1 & 0.999995 & Q8L7G0 & 1 & 0.999994 & Q9LTF7 & 1 & 0.999983 \\
\hline Q8LSZ4 & 1 & 0.999987 & Q8L7L8 & 1 & 0.999986 & Q9FYK5 & 1 & 0.999991 \\
\hline O80452 & 1 & 0.999902 & F4IUG9 & 1 & 0.999864 & P0DH99 & 0 & 4.05E-06 \\
\hline Q38906 & 1 & 0.999952 & Q9C6I6 & 0 & 0.00019 & Q38914 & 1 & 0.999997 \\
\hline Q9LHE9 & 1 & 0.999989 & Q9C641 & 0 & 8.39E-06 & Q9SNB9 & 1 & 0.999669 \\
\hline Q8VY00 & 1 & 0.531791 & P36397 & 1 & 0.855187 & Q9ZNU2 & 1 & 0.999995 \\
\hline O23116 & 1 & 0.999988 & Q9C7B1 & 1 & 0.999994 & Q9SAD4 & 1 & 0.999997 \\
\hline Q9ZTX9 & 1 & 0.999991 & Q9SL41 & 1 & 0.999996 & Q9FNY0 & 1 & 0.999988 \\
\hline Q9M347 & 1 & 0.999975 & O80340 & 1 & 0.999938 & Q9ZTX8 & 1 & 0.999996 \\
\hline Q9LR74 & 1 & 0.999924 & Q9LFQ9 & 1 & 0.999941 & Q9FJA2 & 1 & 0.999996 \\
\hline Q9LMT9 & 1 & 0.999798 & Q9FX25 & 1 & 0.999979 & Q9FWX2 & 1 & 0.999997 \\
\hline Q9ASR1 & 0 & $1.22 \mathrm{E}-05$ & B5X582 & 1 & 0.999885 & Q39191 & 1 & 0.99991 \\
\hline Q93YR9 & 1 & 0.999964 & Q8H1Q2 & 0 & $5.73 \mathrm{E}-06$ & Q9FV71 & 1 & 0.999995 \\
\hline 065041 & 0 & $1.56 \mathrm{E}-06$ & Q8VYA3 & 1 & 0.837936 & Q94JM3 & 1 & 0.99999 \\
\hline Q9FH59 & 1 & 0.999994 & Q9FPR3 & 1 & 0.999993 & P93028 & 1 & 0.999661 \\
\hline 004336 & 1 & 0.999947 & Q9XID4 & 1 & 0.999957 & Q9FNV9 & 1 & 0.999754 \\
\hline Q9STX5 & 1 & 0.999981 & Q9M336 & 0 & 0.000132 & O22921 & 1 & 0.999991 \\
\hline Q9LQE3 & 1 & 0.999981 & Q38850 & 1 & 0.999995 & Q0WL56 & 0 & 4.05E-06 \\
\hline Q9C7W7 & 0 & $7.56 \mathrm{E}-07$ & Q9C519 & 1 & 0.999996 & Q9C5W9 & 1 & 0.999979 \\
\hline Q9ZTC3 & 1 & 0.999991 & Q94CF0 & 1 & 0.998005 & 064700 & 0 & 0.00021 \\
\hline Q9XID3 & 0 & 0.379151 & Q9C7I9 & 1 & 0.999969 & O65508 & 1 & 0.999996 \\
\hline B3LF48 & 0 & 0.00616 & Q9STX2 & 0 & $2.04 \mathrm{E}-06$ & Q94A68 & 0 & 0.020809 \\
\hline Q9C8N7 & 1 & 0.999965 & Q9SPG9 & 1 & 0.999996 & O82653 & 1 & 0.999971 \\
\hline Q9LZI2 & 0 & 0.000243 & Q9LUL2 & 1 & 0.999994 & P93022 & 1 & 0.999992 \\
\hline Q9LXV2 & 1 & 0.999997 & F4IW10 & 0 & $6.44 \mathrm{E}-06$ & Q7X8C5 & 1 & 0.999727 \\
\hline Q8GXQ3 & 1 & 0.52808 & Q9XED8 & 1 & 0.99999 & Q9SSA1 & 1 & 0.999998 \\
\hline Q8LDC8 & 1 & 0.999997 & Q9SA25 & 1 & 0.999995 & & & \\
\hline
\end{tabular}




\begin{tabular}{|c|c|c|c|c|c|c|c|c|}
\hline Mouse & & & & & & & & \\
\hline pro_id & pred_label & proba & pro_id & pred_label & proba & pro_id & pred_label & proba \\
\hline P31266 & 1 & 0.999985 & Q03963 & 0 & 0.485465 & Q60751 & 0 & 0.000128 \\
\hline O35942 & 1 & 0.999993 & Q60855 & 1 & 0.999993 & Q9Z2D6 & 1 & 0.999965 \\
\hline Q9QXB9 & 1 & 0.992734 & Q8BK63 & 0 & 0.025907 & Q8BU40 & 1 & 0.9985 \\
\hline P51448 & 1 & 0.999982 & P60766 & 0 & $1.35 \mathrm{E}-06$ & Q99MU3 & 1 & 0.999982 \\
\hline P08414 & 1 & 0.999714 & A2AI05 & 1 & 0.996906 & P51450 & 1 & 0.999653 \\
\hline Q9R1E0 & 1 & 0.999996 & Q9Z2R9 & 1 & 0.999845 & Q9CXV0 & 1 & 0.805764 \\
\hline Q02780 & 1 & 0.999993 & Q6NTA4 & 1 & 0.997836 & P02340 & 0 & 0.001879 \\
\hline P56931 & 1 & 0.999957 & Q9D2Z8 & 1 & 0.974999 & P97863 & 1 & 0.999996 \\
\hline Q80X95 & 1 & 0.999946 & P00520 & 1 & 0.999993 & Q8R0K9 & 1 & 0.999969 \\
\hline P68181 & 1 & 0.940377 & Q8R4B8 & 1 & 0.999976 & Q99K70 & 0 & $6.76 \mathrm{E}-05$ \\
\hline Q60611 & 1 & 0.999985 & Q61502 & 1 & 0.992386 & Q9DC28 & 1 & 0.991034 \\
\hline Q8C6J9 & 1 & 0.998147 & A2A825 & 0 & 0.020896 & Q3UHJ0 & 1 & 0.999995 \\
\hline Q58FA4 & 1 & 0.999972 & O35066 & 1 & 0.999975 & Q8CIZ9 & 0 & $2.93 E-05$ \\
\hline Q6IMB1 & 0 & $5.29 \mathrm{E}-05$ & Q61102 & 0 & $2.86 \mathrm{E}-06$ & P00375 & 0 & $1.32 \mathrm{E}-05$ \\
\hline P28738 & 1 & 0.999981 & P70255 & 0 & 0.021455 & Q9WTM5 & 1 & 0.938196 \\
\hline P50544 & 0 & 4.14E-05 & O35261 & 1 & 0.999995 & Q9WV04 & 1 & 0.993251 \\
\hline P70257 & 1 & 0.999995 & O35488 & 0 & $5.92 \mathrm{E}-06$ & B5X0E4 & 0 & 0.350486 \\
\hline 054917 & 1 & 0.999988 & P05532 & 0 & 0.002681 & Q60641 & 1 & 0.999947 \\
\hline Q91VE0 & 0 & $1.51 \mathrm{E}-05$ & Q9JJ59 & 0 & $1.21 \mathrm{E}-06$ & Q01279 & 0 & 3.10E-05 \\
\hline Q62120 & 1 & 0.994526 & O88942 & 1 & 0.999993 & Q60714 & 0 & 4.66E-05 \\
\hline Q61271 & 0 & $8.94 \mathrm{E}-06$ & P39053 & 0 & 0.08519 & P05132 & 0 & $6.72 E-05$ \\
\hline Q60591 & 1 & 0.999918 & P60122 & 0 & $1.86 \mathrm{E}-05$ & Q8VHH7 & 0 & $1.42 \mathrm{E}-05$ \\
\hline Q61501 & 1 & 0.999993 & Q8BTH8 & 1 & 0.999959 & Q3UP24 & 0 & 0.00165 \\
\hline Q9JHW4 & 0 & 0.000364 & Q4JIM5 & 1 & 0.999985 & Q6S7F2 & 1 & 0.999979 \\
\hline Q8C4X2 & 0 & $8.58 \mathrm{E}-05$ & Q8K120 & 1 & 0.999928 & Q8VDQ8 & 0 & 0.000751 \\
\hline Q8K440 & 0 & 7.97E-05 & P10126 & 0 & $4.47 \mathrm{E}-06$ & Q52KG5 & 1 & 0.999994 \\
\hline Q99MA9 & 1 & 0.999959 & Q62231 & 1 & 0.999997 & Q61288 & 0 & 0.008277 \\
\hline Q61527 & 0 & $2.28 \mathrm{E}-05$ & Q9QWT9 & 1 & 0.999226 & P42582 & 1 & 0.999957 \\
\hline Q61321 & 1 & 0.99999 & P31750 & 1 & 0.999793 & Q9EQY0 & 0 & 0.095252 \\
\hline P31324 & 0 & $1.23 \mathrm{E}-05$ & Q8BVE8 & 1 & 0.999995 & P70178 & 1 & 0.999967 \\
\hline Q8BXK8 & 1 & 0.999972 & Q03145 & 1 & 0.973974 & Q04447 & 1 & 0.729765 \\
\hline P97305 & 1 & 0.999921 & A2AKX3 & 1 & 0.999992 & P19091 & 0 & 0.000194 \\
\hline Q61772 & 0 & $2.48 \mathrm{E}-05$ & P28867 & 0 & 0.001198 & O54949 & 1 & 0.890301 \\
\hline Q91ZW3 & 1 & 0.999979 & Q8BXL7 & 0 & $1.00 \mathrm{E}-05$ & O09127 & 0 & 0.000173 \\
\hline P68404 & 1 & 0.999508 & P29477 & 0 & 0.00709 & P42208 & 0 & 0.000827 \\
\hline P84078 & 1 & 0.999232 & P54763 & 0 & $1.89 \mathrm{E}-05$ & Q62101 & 0 & 0.001701 \\
\hline Q3UV55 & 1 & 0.999998 & Q923E4 & 1 & 0.999914 & Q8CG76 & 0 & 0.002691 \\
\hline Q8BHK9 & 1 & 0.999941 & Q8BZ03 & 1 & 0.999864 & Q64249 & 1 & 0.999971 \\
\hline Q8R216 & 1 & 0.998369 & Q640N2 & 1 & 0.994561 & Q8CBF3 & 1 & 0.999678 \\
\hline
\end{tabular}




\begin{tabular}{|c|c|c|c|c|c|c|c|c|}
\hline P0C605 & 1 & 0.999091 & Q2LKU9 & 0 & 0.000258 & Q9WVC6 & 0 & 0.000159 \\
\hline P61208 & 0 & $1.32 \mathrm{E}-05$ & P41163 & 1 & 0.983823 & O35071 & 1 & 0.999991 \\
\hline Q91WS2 & 1 & 0.99995 & Q8R104 & 0 & $2.38 \mathrm{E}-05$ & Q99PE9 & 0 & 0.000782 \\
\hline Q8CHI8 & 1 & 0.999978 & Q61768 & 1 & 0.999987 & Q9Z0J4 & 1 & 0.856325 \\
\hline Q8CG48 & 1 & 0.999749 & O54984 & 0 & $7.43 E-05$ & P70424 & 1 & 0.99971 \\
\hline Q02111 & 0 & 0.068938 & Q60644 & 1 & 0.999996 & Q8CG46 & 0 & 0.000828 \\
\hline P70704 & 0 & $2.56 \mathrm{E}-05$ & P81270 & 1 & 0.999974 & P18654 & 0 & 0.001111 \\
\hline P46460 & 0 & $1.47 \mathrm{E}-06$ & Q9CW03 & 1 & 0.999267 & Q925I1 & 1 & 0.99999 \\
\hline P15037 & 1 & 0.999912 & P06151 & 0 & $5.03 E-06$ & O88708 & 1 & 0.999988 \\
\hline Q8CG47 & 1 & 0.999853 & P27040 & 0 & 1.85E-05 & P27577 & 1 & 0.999972 \\
\hline B1AVY7 & 0 & 0.009703 & Q640Q5 & 1 & 0.999936 & Q924W5 & 1 & 0.999152 \\
\hline O70126 & 1 & 0.99999 & P41164 & 1 & 0.999998 & P48025 & 0 & $5.06 \mathrm{E}-05$ \\
\hline Q8CGY8 & 0 & $5.21 \mathrm{E}-06$ & P53783 & 1 & 0.999964 & Q61687 & 1 & 0.999993 \\
\hline P16092 & 0 & 0.021592 & P06240 & 0 & $2.28 \mathrm{E}-05$ & P41778 & 1 & 0.999997 \\
\hline Q9QYY8 & 1 & 0.999905 & Q91YE5 & 1 & 0.999984 & 089106 & 0 & $3.65 \mathrm{E}-05$ \\
\hline Q6ZPI3 & 1 & 0.999994 & P17918 & 0 & 2.39E-05 & P17433 & 1 & 0.999992 \\
\hline P79621 & 1 & 0.99998 & Q8K219 & 1 & 0.99999 & Q8CGK3 & 0 & 0.000395 \\
\hline Q8CIN4 & 0 & 0.001038 & Q62232 & 1 & 0.999997 & P28028 & 1 & 0.999979 \\
\hline P34152 & 1 & 0.99974 & P63006 & 1 & 0.999991 & Q61036 & 1 & 0.997466 \\
\hline P48432 & 1 & 0.999993 & Q5RJI5 & 1 & 0.999945 & Q9QVP9 & 1 & 0.999829 \\
\hline P25911 & 1 & 0.996865 & O88554 & 1 & 0.999953 & Q9CU62 & 1 & 0.999902 \\
\hline 088700 & 1 & 0.99999 & P11276 & 1 & 0.62963 & Q5S006 & 1 & 0.616635 \\
\hline Q8CEE6 & 0 & 0.000125 & Q8Cl15 & 0 & 0.000582 & P36895 & 0 & 0.001602 \\
\hline P16879 & 0 & 0.048174 & Q9WU40 & 1 & 0.999992 & Q3UA06 & 1 & 0.629743 \\
\hline P53784 & 1 & 0.999947 & Q9Z1S0 & 1 & 0.999983 & P35583 & 1 & 0.999995 \\
\hline P49717 & 1 & 0.999957 & P11103 & 1 & 0.999963 & Q811W0 & 1 & 0.999972 \\
\hline P35991 & 0 & $2.49 \mathrm{E}-05$ & Q64732 & 1 & 0.999998 & P97311 & 0 & 0.000261 \\
\hline Q69Z38 & 1 & 0.999989 & P05480 & 1 & 0.673597 & Q69Z98 & 1 & 0.999927 \\
\hline P58462 & 1 & 0.875346 & P08923 & 1 & 0.987073 & Q8CIW5 & 1 & 0.999956 \\
\hline 070551 & 1 & 0.999992 & P49615 & 0 & 5.09E-06 & Q8BPY9 & 1 & 0.999993 \\
\hline Q99MV5 & 1 & 0.99725 & P09041 & 0 & $5.75 \mathrm{E}-06$ & O88697 & 1 & 0.999987 \\
\hline P11440 & 0 & $1.61 \mathrm{E}-05$ & P35582 & 1 & 0.999992 & Q9EQJ9 & 1 & 0.999736 \\
\hline P06803 & 0 & 3.40E-06 & Q80TF6 & 1 & 0.999827 & Q8R3L8 & 0 & $1.01 \mathrm{E}-05$ \\
\hline P35584 & 1 & 0.999987 & Q8VHJ5 & 1 & 0.99994 & Q99LC9 & 1 & 0.999556 \\
\hline Q9WTK7 & 0 & 0.042901 & Q99J95 & 0 & 4.86E-05 & Q9WVH4 & 1 & 0.99998 \\
\hline Q9R1L5 & 1 & 0.999963 & Q07832 & 1 & 0.999579 & O55098 & 0 & 0.001977 \\
\hline P30658 & 1 & 0.999993 & Q61080 & 1 & 0.995941 & P21440 & 0 & 4.68E-06 \\
\hline Q60806 & 1 & 0.999992 & Q8CGC7 & 1 & 0.999271 & Q6A068 & 1 & 0.99997 \\
\hline P16858 & 0 & $1.61 \mathrm{E}-06$ & P49138 & 1 & 0.998727 & P70268 & 1 & 0.999978 \\
\hline P99024 & 1 & 0.643341 & P27790 & 1 & 0.999971 & P06537 & 1 & 0.999947 \\
\hline Q2KHI9 & 1 & 0.999321 & Q64702 & 1 & 0.999995 & Q14BI7 & 1 & 0.999731 \\
\hline P40201 & 1 & 0.99983 & Q3V3I2 & 0 & $5.47 \mathrm{E}-05$ & P14152 & 0 & $7.98 \mathrm{E}-06$ \\
\hline
\end{tabular}




\begin{tabular}{|c|c|c|c|c|c|c|c|c|}
\hline Q9JHG7 & 1 & 0.986799 & O70306 & 1 & 0.999997 & Q9Z265 & 1 & 0.999987 \\
\hline Q8K349 & 0 & 6.80E-06 & P06795 & 0 & 0.000225 & Q99MQ3 & 0 & 4.24E-05 \\
\hline O35144 & 1 & 0.999987 & P26361 & 0 & 0.001353 & P20612 & 0 & 1.39E-05 \\
\hline Q60954 & 1 & 0.999997 & Q922R0 & 0 & 0.000135 & Q01853 & 0 & 0.000253 \\
\hline 070496 & 0 & 2.85E-06 & O70443 & 0 & $3.44 \mathrm{E}-05$ & P97367 & 1 & 0.999998 \\
\hline O88685 & 0 & 0.127826 & Q9EPZ6 & 1 & 0.999995 & P97377 & 0 & 0.000281 \\
\hline P36916 & 1 & 0.999953 & Q8BVN9 & 1 & 0.999992 & Q61136 & 1 & 0.999877 \\
\hline Q8C0Y1 & 1 & 0.999986 & O35491 & 1 & 0.999998 & Q9DC51 & 0 & $6.21 \mathrm{E}-05$ \\
\hline Q63943 & 1 & 0.999994 & P54775 & 0 & $1.03 \mathrm{E}-05$ & O35857 & 1 & 0.999962 \\
\hline A2A8L1 & 1 & 0.999996 & Q6ROH7 & 1 & 0.999932 & Q8CIP4 & 1 & 0.99996 \\
\hline P62196 & 0 & $1.04 \mathrm{E}-05$ & P68372 & 1 & 0.998995 & Q91XQ5 & 0 & 0.000773 \\
\hline P30678 & 0 & 0.006898 & P49718 & 0 & 0.000653 & Q8R4E6 & 1 & 0.999993 \\
\hline Q9CWU0 & 1 & 0.999981 & Q9QUP4 & 0 & 4.51E-06 & P21278 & 0 & 0.00574 \\
\hline P21447 & 0 & $9.62 \mathrm{E}-06$ & Q64434 & 0 & $3.92 \mathrm{E}-06$ & Q64163 & 1 & 0.999983 \\
\hline P22518 & 1 & 0.999995 & P30677 & 0 & $9.25 \mathrm{E}-05$ & Q3UMW7 & 1 & 0.96428 \\
\hline P63011 & 0 & $5.40 \mathrm{E}-06$ & O55047 & 1 & 0.998904 & Q9D5U8 & 1 & 0.777025 \\
\hline P63094 & 1 & 0.739216 & Q9CWV1 & 0 & 0.134263 & O35295 & 1 & 0.999622 \\
\hline Q08639 & 1 & 0.999985 & O35280 & 1 & 0.999967 & Q6PGG6 & 1 & 0.999921 \\
\hline P97310 & 1 & 0.999814 & Q3TMX7 & 0 & 2.04E-05 & A2ASS6 & 1 & 0.953747 \\
\hline Q9R194 & 0 & 6.77E-05 & P20029 & 0 & 0.014554 & P32442 & 1 & 0.999987 \\
\hline Q9CQD1 & 0 & 2.72E-05 & Q01320 & 1 & 0.999996 & P41241 & 0 & $3.74 \mathrm{E}-06$ \\
\hline Q8R316 & 1 & 0.999989 & Q8CFN5 & 1 & 0.999997 & Q99N57 & 1 & 0.999958 \\
\hline Q9ER41 & 0 & 2.53E-06 & P09581 & 0 & 2.17E-05 & P62806 & 0 & 2.17E-06 \\
\hline Q9נI70 & 1 & 0.603738 & P15919 & 1 & 0.99998 & Q64511 & 1 & 0.999993 \\
\hline Q60737 & 1 & 0.999956 & Q9QZR5 & 1 & 0.999997 & Q62504 & 1 & 0.999984 \\
\hline P62492 & 0 & $3.98 \mathrm{E}-05$ & Q8CGV9 & 1 & 0.999998 & P97784 & 1 & 0.958231 \\
\hline P70349 & 0 & 1.10E-05 & Q9WTU6 & 0 & 1.83E-05 & P56371 & 0 & 0.000689 \\
\hline Q68FE9 & 1 & 0.999972 & O70589 & 1 & 0.998168 & P30681 & 1 & 0.999995 \\
\hline Q9WUI1 & 0 & 0.002091 & P63001 & 0 & 2.07E-06 & Q3UVR3 & 1 & 0.999816 \\
\hline P56546 & 0 & 0.01567 & O54879 & 1 & 0.999991 & Q9Z1B7 & 0 & 0.022377 \\
\hline E9Q9D5 & 1 & 0.995724 & Q80UG8 & 1 & 0.999005 & P53564 & 1 & 0.999981 \\
\hline Q8R1H0 & 1 & 0.999526 & Q8BG51 & 1 & 0.99921 & 008674 & 1 & 0.999898 \\
\hline A2APC3 & 1 & 0.999978 & O54833 & 1 & 0.999959 & P17095 & 0 & $1.73 \mathrm{E}-05$ \\
\hline P47811 & 0 & $5.74 \mathrm{E}-06$ & Q08297 & 0 & 8.17E-06 & Q925K9 & 0 & 0.000104 \\
\hline Q8VDF3 & 0 & 0.005746 & P42581 & 1 & 0.999993 & Q8CE90 & 1 & 0.84386 \\
\hline Q61411 & 0 & 3.81E-05 & Q02053 & 1 & 0.997363 & Q91VR5 & 1 & 0.981173 \\
\hline P17156 & 0 & 1.16E-05 & Q7TT50 & 0 & 5.95E-05 & P32883 & 0 & 0.000141 \\
\hline Q3U3Q1 & 0 & 0.003266 & Q62167 & 1 & 0.999965 & P63017 & 0 & $6.80 \mathrm{E}-06$ \\
\hline Q9WUI0 & 1 & 0.999994 & Q8VEA8 & 0 & 2.36E-06 & Q80X41 & 1 & 0.999969 \\
\hline Q61496 & 1 & 0.99998 & Q61696 & 0 & 1.70E-05 & P23249 & 0 & 0.022272 \\
\hline P43352 & 1 & 0.999995 & Q8BN21 & 1 & 0.999979 & Q61655 & 1 & 0.993361 \\
\hline P63158 & 1 & 0.999993 & Q9R1X5 & 0 & 8.73E-06 & Q62193 & 1 & 0.99999 \\
\hline
\end{tabular}




\begin{tabular}{|c|c|c|c|c|c|c|c|c|}
\hline Q8VEJ9 & 1 & 0.999982 & Q9JJY4 & 1 & 0.999956 & P17879 & 0 & $1.82 \mathrm{E}-05$ \\
\hline Q91Y86 & 0 & $2.23 \mathrm{E}-05$ & P08556 & 0 & 0.000497 & P46467 & 1 & 0.99998 \\
\hline O70133 & 1 & 0.999902 & P16627 & 0 & 1.83E-05 & 008911 & 0 & $9.21 \mathrm{E}-06$ \\
\hline P35546 & 0 & 0.000115 & Q8VCS3 & 0 & $6.94 \mathrm{E}-06$ & Q9ESV0 & 0 & 0.000375 \\
\hline P09022 & 1 & 0.999986 & Q99MT2 & 1 & 0.999991 & P35601 & 1 & 0.999985 \\
\hline P70121 & 1 & 0.999966 & Q810A7 & 1 & 0.99882 & Q91VC3 & 1 & 0.9982 \\
\hline Q9R1S7 & 0 & $7.11 \mathrm{E}-06$ & Q9WUK4 & 0 & $1.67 \mathrm{E}-06$ & P43404 & 1 & 0.999945 \\
\hline Q8BG36 & 1 & 0.999953 & Q60680 & 1 & 0.999589 & P13705 & 1 & 0.999836 \\
\hline Q99J62 & 0 & $8.78 \mathrm{E}-06$ & Q9R0G7 & 1 & 0.999995 & Q99J87 & 0 & 0.220782 \\
\hline O88351 & 1 & 0.89347 & Q61006 & 0 & $1.90 \mathrm{E}-05$ & P48377 & 1 & 0.999981 \\
\hline Q8C0C0 & 1 & 0.99999 & P70397 & 1 & 0.999997 & Q62406 & 1 & 0.999978 \\
\hline Q64331 & 1 & 0.999921 & P48379 & 0 & 0.017989 & Q6NZP1 & 1 & 0.996623 \\
\hline Q61880 & 0 & $1.75 \mathrm{E}-05$ & Q9R0T8 & 0 & $2.09 \mathrm{E}-05$ & Q02566 & 0 & 0.288783 \\
\hline Q8VEE4 & 1 & 0.999927 & E9PZI6 & 1 & 0.999449 & Q8BYH0 & 1 & 0.999975 \\
\hline P81067 & 1 & 0.999982 & P09922 & 1 & 0.999976 & P48381 & 1 & 0.999928 \\
\hline H3BKF3 & 1 & 0.999922 & Q9JHU4 & 0 & 0.000152 & P70671 & 0 & 0.480628 \\
\hline Q8VDD5 & 1 & 0.998454 & Q9D0F6 & 0 & $6.50 \mathrm{E}-05$ & E9QKG2 & 1 & 0.999869 \\
\hline P70396 & 1 & 0.999998 & P15208 & 1 & 0.99942 & P97479 & 1 & 0.999914 \\
\hline P62746 & 0 & $8.09 \mathrm{E}-06$ & & & & & & \\
\hline \multicolumn{9}{|l|}{ S.cerevisiae } \\
\hline pro_id & pred_label & proba & pro_id & pred_label & proba & pro_id & pred_label & proba \\
\hline Q12154 & 1 & 0.96329 & P16521 & 0 & 0.000414 & P28742 & 1 & 0.999994 \\
\hline P07272 & 1 & 0.999994 & P13574 & 1 & 0.999991 & P22336 & 1 & 0.999993 \\
\hline P36041 & 1 & 0.999997 & P38691 & 1 & 0.999992 & P33298 & 0 & 0.000273 \\
\hline Q03497 & 1 & 0.999996 & P11938 & 1 & 0.999997 & P32324 & 0 & 5.33E-06 \\
\hline Q06147 & 1 & 0.999991 & Q01939 & 0 & $1.37 \mathrm{E}-05$ & P12866 & 0 & $7.72 \mathrm{E}-06$ \\
\hline P06786 & 1 & 0.999996 & P32476 & 0 & $2.74 \mathrm{E}-06$ & P13186 & 1 & 0.999983 \\
\hline P53131 & 1 & 0.996826 & P32916 & 1 & 0.999968 & P25847 & 1 & 0.999962 \\
\hline P38071 & 1 & 0.999991 & P36004 & 1 & 0.999988 & P33299 & 0 & 0.004518 \\
\hline P38699 & 1 & 0.999996 & P08539 & 0 & 1.03E-05 & P38137 & 1 & 0.994192 \\
\hline P34253 & 0 & 0.06208 & P20095 & 1 & 0.999695 & P36057 & 0 & 9.39E-06 \\
\hline P01123 & 0 & $1.78 \mathrm{E}-05$ & P35731 & 1 & 0.604415 & Q12246 & 1 & 0.999951 \\
\hline P53549 & 0 & 0.000333 & P32597 & 1 & 0.999217 & P26754 & 1 & 0.999983 \\
\hline P41813 & 1 & 0.999996 & P53145 & 1 & 0.998181 & P40327 & 0 & 0.002121 \\
\hline P25302 & 1 & 0.999998 & P07271 & 1 & 0.99996 & Q04433 & 1 & 0.999979 \\
\hline P36775 & 0 & 0.08869 & P21372 & 1 & 0.998372 & P04802 & 1 & 0.594045 \\
\hline P06780 & 1 & 0.999846 & P00360 & 0 & 1.15E-06 & P30665 & 1 & 0.999994 \\
\hline P33297 & 0 & 0.000171 & Q07478 & 0 & 4.73E-05 & Q03834 & 1 & 0.999995 \\
\hline P00359 & 0 & $1.63 \mathrm{E}-06$ & P29496 & 1 & 0.999975 & P47116 & 1 & 0.999984 \\
\hline P08153 & 1 & 0.999995 & P40433 & 1 & 0.999956 & P00358 & 0 & $8.50 \mathrm{E}-06$ \\
\hline
\end{tabular}




\begin{tabular}{|c|c|c|c|c|c|c|c|c|}
\hline P53091 & 1 & 0.999977 & P31374 & 1 & 0.997852 & P32944 & 1 & 0.999987 \\
\hline P39925 & 0 & $2.85 \mathrm{E}-05$ & P14065 & 1 & 0.999977 & P29469 & 1 & 0.999918 \\
\hline P41909 & 1 & 0.958429 & P40340 & 1 & 0.999995 & P00330 & 0 & 5.07E-06 \\
\hline P43535 & 1 & 0.998595 & P38132 & 1 & 0.999984 & P34230 & 1 & 0.999958 \\
\hline Q02457 & 1 & 0.999966 & P00331 & 0 & 7.31E-06 & Q12263 & 1 & 0.999991 \\
\hline Q12019 & 0 & 0.34795 & P80210 & 0 & $7.12 \mathrm{E}-05$ & P18412 & 1 & 0.999997 \\
\hline P43633 & 1 & 0.999995 & P15442 & 1 & 0.999897 & 014467 & 1 & 0.998451 \\
\hline P31244 & 1 & 0.999998 & P36145 & 1 & 0.99999 & P25377 & 0 & $1.83 \mathrm{E}-06$ \\
\hline Q08726 & 0 & $5.16 \mathrm{E}-06$ & P36060 & 0 & 2.79E-06 & P12753 & 0 & 0.430918 \\
\hline P42938 & 0 & 0.000151 & P46367 & 0 & $2.78 \mathrm{E}-06$ & P32836 & 0 & 3.17E-05 \\
\hline P33310 & 0 & $5.31 \mathrm{E}-06$ & P25454 & 0 & 0.037007 & Q03533 & 0 & 0.011192 \\
\hline P54115 & 0 & $8.04 \mathrm{E}-07$ & P32835 & 0 & $2.15 \mathrm{E}-05$ & P24279 & 1 & 0.99998 \\
\hline P38953 & 0 & 0.00034 & P09733 & 0 & $1.85 \mathrm{E}-06$ & P32789 & 1 & 0.999988 \\
\hline P02309 & 0 & $4.71 \mathrm{E}-06$ & P33311 & 0 & 0.000113 & P01119 & 1 & 0.978933 \\
\hline P09734 & 0 & $2.08 \mathrm{E}-06$ & P38116 & 0 & $8.23 \mathrm{E}-05$ & P47122 & 1 & 0.996396 \\
\hline P07884 & 1 & 0.999985 & P39729 & 0 & $5.31 \mathrm{E}-05$ & Q01852 & 1 & 0.999953 \\
\hline Q02804 & 1 & 0.989574 & Q03532 & 1 & 0.999947 & P19358 & 0 & $2.25 \mathrm{E}-05$ \\
\hline P19541 & 1 & 0.999998 & P23254 & 0 & $1.65 \mathrm{E}-06$ & P32381 & 0 & $1.29 \mathrm{E}-05$ \\
\hline P10823 & 1 & 0.999776 & P53166 & 0 & 0.21253 & P14736 & 1 & 0.999996 \\
\hline P22515 & 1 & 0.999345 & P11076 & 1 & 0.998987 & P41911 & 1 & 0.999591 \\
\hline P32333 & 1 & 0.999809 & P22216 & 1 & 0.999957 & P52488 & 0 & 0.000181 \\
\hline P05085 & 1 & 0.999992 & P42950 & 1 & 0.998909 & P25336 & 1 & 0.999994 \\
\hline P32863 & 1 & 0.999971 & P38820 & 0 & 0.000144 & Q12386 & 1 & 0.999993 \\
\hline P38970 & 0 & 0.000501 & P47047 & 0 & 0.003943 & P32849 & 0 & 0.071631 \\
\hline P26370 & 1 & 0.999997 & P53974 & 1 & 0.999989 & P25364 & 1 & 0.99999 \\
\hline P25846 & 1 & 0.999974 & P38623 & 1 & 0.999856 & P38735 & 0 & 0.000387 \\
\hline P49090 & 0 & 0.00578 & P51979 & 1 & 0.999955 & P47069 & 1 & 0.999176 \\
\hline P06838 & 1 & 0.999998 & P18562 & 0 & 0.000389 & P40467 & 1 & 0.999995 \\
\hline POCE41 & 1 & 0.999994 & Q05473 & 1 & 0.99984 & P01120 & 0 & $1.50 \mathrm{E}-05$ \\
\hline P22219 & 1 & 0.928891 & P53104 & 0 & 0.015727 & Q12753 & 1 & 0.999998 \\
\hline P0CY06 & 1 & 0.999994 & P53879 & 1 & 0.999872 & P21576 & 0 & 0.031375 \\
\hline P40416 & 0 & $1.77 \mathrm{E}-05$ & P34243 & 1 & 0.99999 & P28737 & 0 & 0.001066 \\
\hline Q08904 & 1 & 0.999981 & Q04500 & 1 & 0.999805 & P40471 & 0 & $6.41 \mathrm{E}-06$ \\
\hline Q04458 & 0 & 0.001508 & P0CY08 & 1 & 0.999977 & P25611 & 1 & 0.99981 \\
\hline Q06685 & 0 & 0.110151 & Q01389 & 1 & 0.999993 & P0CY07 & 1 & 0.999994 \\
\hline P53128 & 0 & $2.04 \mathrm{E}-05$ & Q03195 & 0 & $2.27 \mathrm{E}-05$ & P52917 & 1 & 0.936013 \\
\hline P14772 & 0 & $9.88 \mathrm{E}-05$ & P0CY11 & 1 & 0.999995 & P32492 & 1 & 0.999792 \\
\hline P38766 & 1 & 0.999993 & P36017 & 0 & 0.415801 & P32639 & 1 & 0.999408 \\
\hline P02829 & 1 & 0.999847 & Q04439 & 1 & 0.999984 & P38712 & 0 & 0.000681 \\
\hline P39961 & 1 & 0.999992 & P43569 & 0 & $3.12 \mathrm{E}-06$ & P34244 & 1 & 0.99999 \\
\hline P38626 & 0 & $3.28 \mathrm{E}-06$ & Q01163 & 1 & 0.898718 & Q12222 & 0 & 0.000167 \\
\hline P23293 & 1 & 0.999967 & P32485 & 0 & 7.60E-06 & P19524 & 1 & 0.99998 \\
\hline
\end{tabular}




\begin{tabular}{|c|c|c|c|c|c|c|c|c|}
\hline Q12464 & 0 & 0.000128 & P14680 & 1 & 0.999996 & Q03941 & 1 & 0.994428 \\
\hline P33416 & 1 & 0.999812 & P36006 & 1 & 0.999975 & P38990 & 1 & 0.999924 \\
\hline P39109 & 0 & 4.79E-05 & P32468 & 1 & 0.999981 & P0CY09 & 1 & 0.999977 \\
\hline P38830 & 1 & 0.999984 & P20606 & 0 & $1.71 \mathrm{E}-06$ & P36086 & 0 & $9.02 \mathrm{E}-06$ \\
\hline P27636 & 1 & 0.989915 & P31539 & 0 & $1.49 \mathrm{E}-05$ & P16603 & 0 & 0.006093 \\
\hline P39954 & 0 & $7.29 \mathrm{E}-07$ & Q12152 & 1 & 0.999988 & P32457 & 1 & 0.999981 \\
\hline Q08732 & 1 & 0.999996 & P25293 & 1 & 0.999832 & P18759 & 0 & 7.77E-06 \\
\hline P12688 & 1 & 0.999983 & P25342 & 1 & 0.995706 & Q05549 & 1 & 0.947624 \\
\hline Q03435 & 1 & 0.999963 & P39955 & 1 & 0.99997 & P53049 & 1 & 0.973036 \\
\hline P32458 & 0 & 0.152439 & P32481 & 0 & $6.28 \mathrm{E}-06$ & P52920 & 0 & 0.000116 \\
\hline P11792 & 1 & 0.999998 & P48559 & 1 & 0.999977 & P09119 & 1 & 0.999701 \\
\hline P53685 & 1 & 0.999971 & Q08972 & 1 & 0.999785 & P46954 & 1 & 0.99999 \\
\hline Q99260 & 1 & 0.99998 & P40558 & 1 & 0.999986 & P10081 & 0 & 0.000547 \\
\hline P11633 & 0 & $1.14 \mathrm{E}-05$ & Q08491 & 1 & 0.998335 & P43591 & 1 & 0.999901 \\
\hline P19073 & 0 & $7.54 \mathrm{E}-07$ & P06168 & 1 & 0.786166 & P36003 & 1 & 0.999996 \\
\hline Q99287 & 0 & 0.034375 & P38146 & 0 & 0.000123 & P25694 & 0 & $2.36 \mathrm{E}-06$ \\
\hline P38697 & 0 & $8.90 \mathrm{E}-05$ & Q12181 & 1 & 0.997511 & P07560 & 0 & $1.34 \mathrm{E}-06$ \\
\hline P51996 & 0 & 0.000286 & P32797 & 1 & 0.999985 & P04807 & 0 & $1.40 \mathrm{E}-05$ \\
\hline P11632 & 1 & 0.908247 & Q00416 & 1 & 0.999982 & P36018 & 0 & 0.004907 \\
\hline P32562 & 1 & 0.604062 & P53115 & 1 & 0.999909 & P38219 & 0 & 0.000305 \\
\hline Q07657 & 1 & 0.9916 & P32939 & 0 & $2.74 \mathrm{E}-05$ & P22516 & 1 & 0.999966 \\
\hline P38991 & 1 & 0.981133 & P40010 & 1 & 0.999973 & P35207 & 1 & 0.999942 \\
\hline Q04116 & 1 & 0.999995 & Q03654 & 1 & 0.999985 & P05986 & 0 & 0.000257 \\
\hline P39720 & 1 & 0.999988 & P32908 & 1 & 0.999967 & Q12244 & 1 & 0.999969 \\
\hline P32657 & 1 & 0.999961 & P32361 & 1 & 0.977522 & P36023 & 1 & 0.999979 \\
\hline P47037 & 1 & 0.999979 & P34161 & 1 & 0.999996 & P27895 & 1 & 0.999978 \\
\hline P17119 & 1 & 0.998927 & P15873 & 1 & 0.999527 & P06700 & 1 & 0.999923 \\
\hline P40341 & 1 & 0.998022 & P27697 & 1 & 0.999068 & P39962 & 1 & 0.723849 \\
\hline P13259 & 1 & 0.999995 & Q03656 & 1 & 0.999997 & P38555 & 0 & 0.001263 \\
\hline P15790 & 1 & 0.999987 & P22517 & 0 & 0.000874 & P29468 & 1 & 0.999466 \\
\hline P36048 & 1 & 0.713942 & P19454 & 1 & 0.999891 & Q06554 & 1 & 0.999441 \\
\hline P38126 & 0 & 0.00028 & P35187 & 1 & 0.999833 & P22204 & 1 & 0.999952 \\
\hline P23292 & 1 & 0.999997 & P33751 & 1 & 0.507383 & P20424 & 0 & 5.09E-06 \\
\hline P24783 & 1 & 0.999946 & P07278 & 1 & 0.999985 & P08018 & 1 & 0.999937 \\
\hline P38128 & 1 & 0.999996 & P32328 & 1 & 0.999985 & P15454 & 1 & 0.999907 \\
\hline P12383 & 1 & 0.999997 & P39073 & 1 & 0.999963 & P20449 & 0 & 4.63E-05 \\
\hline P28743 & 1 & 0.999895 & P33302 & 0 & 4.05E-05 & P32364 & 1 & 0.999993 \\
\hline P06634 & 0 & 0.000251 & P23291 & 1 & 0.999996 & P00560 & 0 & $1.90 \mathrm{E}-05$ \\
\hline P22082 & 1 & 0.999992 & P20448 & 1 & 0.99889 & P25389 & 1 & 0.99998 \\
\hline P17157 & 0 & 0.002058 & P41901 & 1 & 0.999996 & P39009 & 1 & 0.999995 \\
\hline P14681 & 0 & 3.23E-05 & P16862 & 0 & 0.000487 & P25808 & 1 & 0.999817 \\
\hline P09624 & 0 & $6.99 \mathrm{E}-07$ & P13185 & 1 & 0.999989 & P52960 & 1 & 0.999983 \\
\hline
\end{tabular}




\begin{tabular}{|c|c|c|c|c|c|c|c|c|}
\hline P12954 & 1 & 0.999992 & P02994 & 0 & $3.92 \mathrm{E}-06$ & P06242 & 0 & 0.000581 \\
\hline Q12236 & 1 & 0.999994 & P50104 & 1 & 0.999997 & & & \\
\hline & & & & & & & & \\
\hline \multicolumn{9}{|l|}{ Fruit fly } \\
\hline pro_id & pred_label & proba & pro_id & pred_label & proba & pro_id & pred_label & proba \\
\hline P15348 & 1 & 0.999994 & A4VCL2 & 1 & 0.999982 & P51023 & 1 & 0.999995 \\
\hline P22058 & 1 & 0.999978 & Q9VGW6 & 0 & $3.48 \mathrm{E}-05$ & P28159 & 1 & 0.999991 \\
\hline Q8SWV6 & 0 & 0.00517 & P52304 & 1 & 0.999987 & P08181 & 0 & $1.64 \mathrm{E}-05$ \\
\hline Q9V461 & 0 & 0.000777 & Q24492 & 0 & 0.000167 & P18106 & 0 & 0.000608 \\
\hline P48601 & 1 & 0.752976 & Q24210 & 0 & 0.004888 & Q00449 & 0 & 0.000676 \\
\hline P23625 & 1 & 0.999623 & P02835 & 1 & 0.999997 & O18413 & 0 & 0.012199 \\
\hline Q9VBW6 & 1 & 0.999995 & P24785 & 0 & $9.95 \mathrm{E}-05$ & P16378 & 0 & 8.03E-05 \\
\hline P25157 & 1 & 0.999982 & P08646 & 0 & $6.96 \mathrm{E}-05$ & Q9VBW9 & 1 & 0.99995 \\
\hline P04197 & 1 & 0.999998 & Q7KU24 & 1 & 0.999969 & Q8MT06 & 0 & 0.001507 \\
\hline P08645 & 0 & $1.42 \mathrm{E}-05$ & Q9VCU9 & 1 & 0.999962 & Q9W3C1 & 1 & 0.999492 \\
\hline P20353 & 0 & $1.85 \mathrm{E}-05$ & Q05337 & 1 & 0.992517 & O18334 & 0 & 0.001639 \\
\hline O76324 & 1 & 0.999855 & Q27571 & 1 & 0.999074 & Q9W252 & 1 & 0.99798 \\
\hline Q9VEI9 & 1 & 0.999996 & P04388 & 0 & 0.084765 & P19109 & 1 & 0.999985 \\
\hline P10676 & 1 & 0.999878 & P20354 & 0 & 0.00303 & Q02748 & 1 & 0.998724 \\
\hline P35600 & 1 & 0.999979 & P23128 & 0 & 0.013709 & P54351 & 0 & 1.06E-05 \\
\hline P00522 & 1 & 0.999982 & Q24208 & 0 & $3.71 \mathrm{E}-06$ & P53034 & 0 & $1.37 \mathrm{E}-05$ \\
\hline Q24533 & 1 & 0.999993 & Q9VPD2 & 0 & 4.96E-06 & P29673 & 1 & 0.999992 \\
\hline Q02280 & 0 & 0.000282 & Q94523 & 0 & 2.12E-05 & P07548 & 1 & 0.999997 \\
\hline P46461 & 0 & $2.82 \mathrm{E}-05$ & P45888 & 0 & $5.15 \mathrm{E}-06$ & Q9V3I5 & 1 & 0.999995 \\
\hline P30052 & 1 & 0.999996 & P49762 & 1 & 0.999994 & Q9VJI9 & 1 & 0.993645 \\
\hline P40945 & 1 & 0.998803 & Q9V4L4 & 0 & $2.98 \mathrm{E}-06$ & Q27324 & 0 & $1.29 \mathrm{E}-05$ \\
\hline P23023 & 1 & 0.999992 & P16554 & 1 & 0.999983 & P40946 & 0 & $2.43 \mathrm{E}-06$ \\
\hline P54367 & 0 & $2.04 \mathrm{E}-05$ & Q9VWQ2 & 1 & 0.999934 & O77051 & 1 & 0.999993 \\
\hline Q95RR8 & 1 & 0.999962 & Q9VW15 & 1 & 0.999995 & P81900 & 1 & 0.999985 \\
\hline Q9VK34 & 1 & 0.999993 & P11536 & 1 & 0.999996 & P31368 & 1 & 0.999991 \\
\hline Q7JWD3 & 0 & $2.61 \mathrm{E}-06$ & P83099 & 0 & 0.000107 & P32031 & 1 & 0.999996 \\
\hline P08736 & 0 & $2.69 \mathrm{E}-06$ & P35875 & 1 & 0.999974 & P35381 & 0 & $2.69 \mathrm{E}-05$ \\
\hline P05130 & 1 & 0.99988 & Q9VL52 & 1 & 0.999959 & Q27368 & 1 & 0.999998 \\
\hline P17917 & 0 & $1.70 \mathrm{E}-05$ & Q05825 & 0 & 4.79E-06 & P13678 & 0 & $3.58 \mathrm{E}-05$ \\
\hline P32030 & 1 & 0.999998 & P20105 & 1 & 0.999995 & 097143 & 0 & 0.078781 \\
\hline Q9W0K4 & 1 & 0.999987 & P91660 & 0 & 0.0064 & Q9VF26 & 1 & 0.999954 \\
\hline P45447 & 1 & 0.99999 & P09081 & 1 & 0.999998 & P17210 & 1 & 0.999986 \\
\hline Q9U5L1 & 0 & 0.066107 & P40427 & 1 & 0.999983 & P40797 & 1 & 0.999897 \\
\hline Q9VC57 & 0 & 0.171307 & P46863 & 1 & 0.999987 & P28668 & 1 & 0.99996 \\
\hline Q9VM92 & 1 & 0.99628 & Q9VSJ5 & 1 & 0.992536 & Q9W0K7 & 1 & 0.999993 \\
\hline O61267 & 1 & 0.99848 & P06606 & 0 & $9.70 \mathrm{E}-05$ & P09052 & 0 & $4.11 \mathrm{E}-05$ \\
\hline
\end{tabular}




\begin{tabular}{|c|c|c|c|c|c|c|c|c|}
\hline A8JNJ2 & 1 & 0.999918 & Q9VGI8 & 1 & 0.999988 & Q9W590 & 1 & 0.985838 \\
\hline P06603 & 0 & $2.60 E-06$ & P20153 & 1 & 0.999966 & Q9VZV3 & 1 & 0.999914 \\
\hline Q9VNP5 & 1 & 0.998937 & Q7KUT2 & 1 & 0.999898 & P61857 & 0 & 0.000234 \\
\hline Q9VA38 & 1 & 0.999997 & P08630 & 1 & 0.998641 & Q9VNS0 & 1 & 0.999995 \\
\hline P06605 & 0 & $2.06 E-06$ & Q95T10 & 0 & 0.151901 & P23573 & 0 & 0.000617 \\
\hline Q26454 & 1 & 0.999919 & P06604 & 0 & $2.73 E-06$ & P28167 & 1 & 0.999996 \\
\hline Q9VT57 & 0 & $2.32 E-05$ & Q00748 & 0 & 0.000565 & Q7KN62 & 0 & $8.32 E-05$ \\
\hline P28166 & 1 & 0.999998 & Q9NGC3 & 1 & 0.99999 & P49735 & 0 & 0.012666 \\
\hline O77277 & 1 & 0.999955 & Q7KV83 & 1 & 0.999926 & O16102 & 1 & 0.999994 \\
\hline Q9XYU1 & 1 & 0.999241 & Q9NBK5 & 0 & 0.000466 & Q8IRE7 & 1 & 0.999913 \\
\hline O77059 & 0 & $2.94 E-05$ & Q9XYU0 & 1 & 0.99998 & P20659 & 1 & 0.999988 \\
\hline Q917T8 & 1 & 0.999878 & & & & & & \\
\hline
\end{tabular}




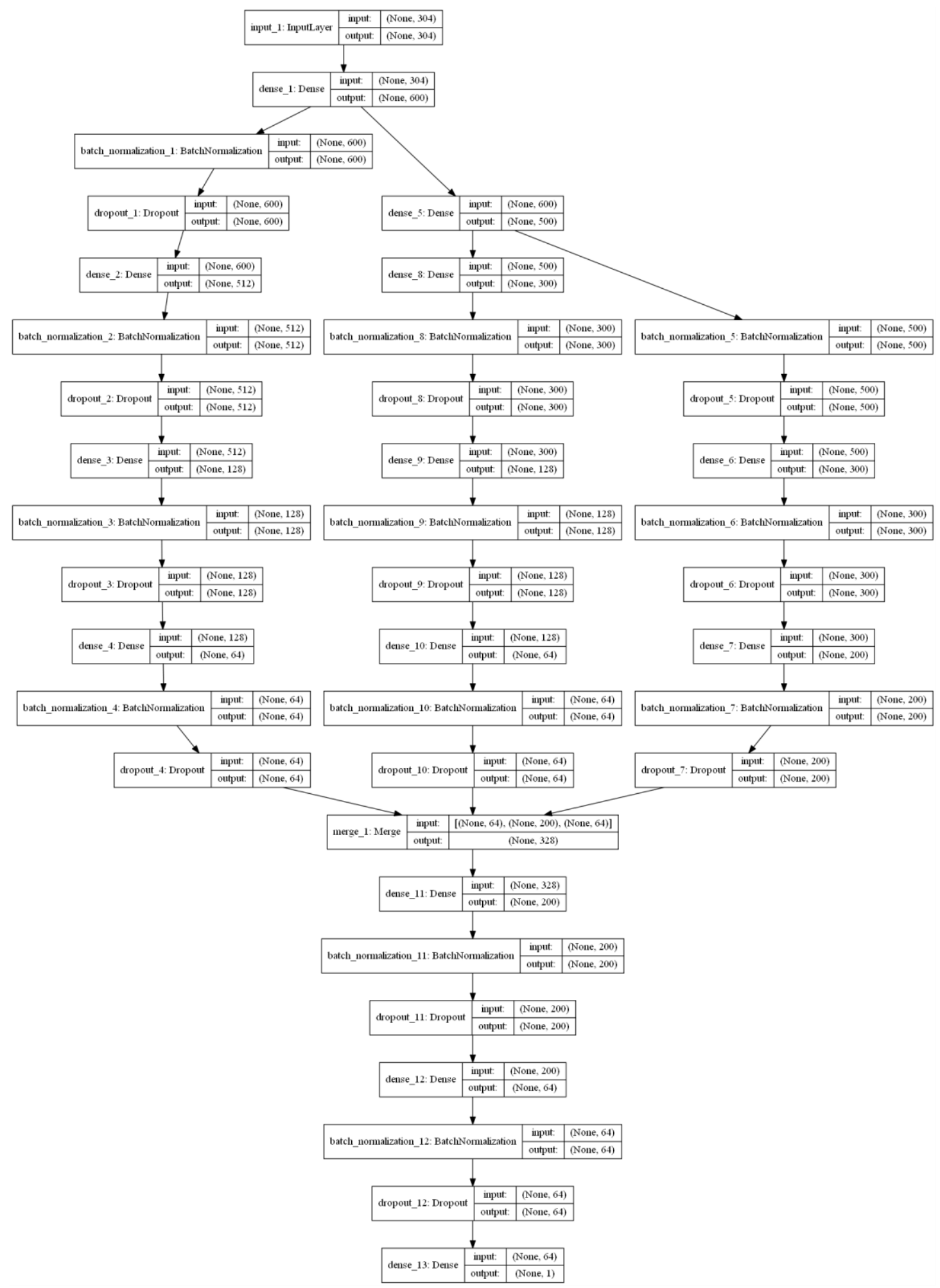

Figure S1. Parameter settings for the model trained on reduced PDB1075. 